3 Jianqin Hao ${ }^{1, \#}$, Dekai Wang ${ }^{2, \#}$, Yingbao Wu ${ }^{1, \#}$, Ke Huang ${ }^{1,6 \#}$, Penggen Duan ${ }^{1}$, Na

4 5 Inzé (,5, $^{\text {, Qian Qian }}{ }^{3^{*}}$ and Yunhai $\mathrm{Li}^{1,6^{*}}$

$6 \quad{ }^{1}$ State Key Laboratory of Plant Cell and Chromosome Engineering, CAS Centre for

\section{The GW2-WG1-OsbZIP47 pathway controls grain size and weight in}

\author{
rice
}

$\mathrm{Li}^{1}$, Ran Xu ${ }^{1}$, Dali Zeng ${ }^{3}$, Guojun Dong ${ }^{3}$, Baolan Zhang ${ }^{1}$, Limin Zhang ${ }^{1}$, Dirk Excellence in Molecular Plant Biology, Institute of Genetics and Developmental Biology, The Innovative Academy of Seed Design, Chinese Academy of Sciences, Beijing, 100101, China.

${ }^{2}$ College of life sciences and medicine, Zhejiang Sci-Tech university, Hangzhou, 310018, China.

${ }^{3}$ State Key Laboratory of Rice Biology, China National Rice Research Institute, Hangzhou, 310006, China.

${ }^{4}$ Ghent University, Department of Plant Biotechnology and Bioinformatics, 9052 Ghent, Belgium.

${ }^{5}$ VIB Center for Plant Systems Biology, 9052 Ghent, Belgium.

${ }^{6}$ University of Chinese Academy of Sciences, Beijing, 100039, China.

${ }^{\#}$ These authors contributed equally to this work.

*Corresponding authors: E-MAIL: yhli@genetics.ac.cn; or qianqian188@hotmail.com.

Running title: The GW2-WG1-OsbZIP47 module regulates grain size.

Key words: rice, WG1, GW2, OsbZIP47, grain size and weight, cell proliferation 3

Short summary: Grain size is one of important yield traits and also determines appearance quality in rice. This study reveals a genetic and molecular framework for the GW2-WG1-OsbZIP47- mediated control of grain size and weight in rice and provides a promising target for genetic improvement of grain yield and appearance quality in key crops. 


\section{Abstract}

Regulation of seed size is a key strategy for improving crop yield, and is also a basic biological question, but how plants determine their seed size remains elusive. Here we report that the GW2-WG1-OsbZIP47 regulatory module controls grain width and weight in rice. WG1, which encodes a glutaredoxin protein, promotes grain growth by increasing cell proliferation. WG1 interacts with the transcription factor OsbZIP47 and represses its transcriptional activity by associating with the transcriptional co-repressor ASP1, indicating that WG1 may act as adaptor protein to recruit the transcriptional co-repressor. OsbZIP47 restricts grain growth by decreasing cell proliferation. Further results reveal that the E3 ubiquitin ligase GW2 ubiquitinates WG1 and targets it for degradation. Genetic analyses support that $G W 2$, WG1 and OsbZIP47 function in a common pathway to control grain growth. Thus, our findings reveal a genetic and molecular framework for the GW2-WG1-OsbZIP47 regulatory module-mediated control of grain size and weight, opening new perspectives for using this regulatory pathway for improvement of seed size and weight in crops. 


\section{Introduction}

Rice is one of the most important food crops worldwide. Increasing rice yield is crucial for supporting the rapid growth of global population (Zhang, 2007). Grain size is a key yield trait and also influences grain appearance quality in rice. The size of a grain is predominantly determined by the grain hull that limits grain growth in rice ( $\mathrm{Li}$ et al., 2019). Several factors that control grain size by influencing cell proliferation in the grain hull have been reported in rice. For example, the G protein $\gamma$ subunit GS3 restricts grain growth by influencing cell proliferation in the grain hull (Fan et al., 2006; Mao et al., 2010). GS3 functions antagonistically with other G $\gamma$ subunits DEP1 and GGC2 to regulate grain length by competitively binding RGB1 (Sun et al., 2018), In contrast, DEP1 has also been reported to repress grain growth by interacting with RGB1 (Liu et al., 2018). The OsMKKK10-OsMKK4-OsMAPK6 cassette regulates grain length by increasing cell proliferation in the grain hull (Duan et al., 2014; Xu et al., 2018a), and OsMKP1 can dephosphorylate MAPK6 to restrict grain growth (Guo et al., 2018; Xu et al., 2018b). The transcriptional regulator GS9, the transcription factor OsMADS1 and the protein phosphatase GL3-1/GL3.1/OsPPKL1 have been reported to control grain length by regulating cell proliferation in the grain hull (Hu et al., 2012; Liu et al., 2018; Qi et al., 2012; Zhang et al., 2012; Zhao et al., 2018), while the E3 ubiquitin ligase GW2, the calmodulin-binding protein GSE5/GW5, and the transcription factor GW8/OsSPL16 have been described to determine grain width by influencing cell proliferation in the grain hull (Duan et al., 2017; Liu et al., 2017; Song et al., 2007; Wang et al., 2012). By contrast, several regulators that regulate grain size by influencing cell expansion in the grain hull have also been reported in rice. The transcription factor OsSPL13 regulates expression of SRS5 and promotes grain growth by influencing cell expansion (Si et al., 2016). The transcription factor GS2/OsGRF4 interacts with the co-activator OsGIF1/2/3 to control grain size predominantly by increasing cell expansion in the grain hull (Che et al., 2015; Duan et al., 2015; Hu et al., 2015). The SHAGGY-like kinase GSK2 phosphorylates RNA-binding protein OML4 to restrict grain growth by influencing cell expansion in the grain hull (Lyu et al., 2020). The SHAGGY-like kinase GSK5 and the TON1 
RECRUITING MOTIF-containing protein GL7 have also been described to regulate grain size by influencing cell expansion in the grain hull (Hu et al., 2018; Wang et al., 2015; Xia et al., 2018; Ying et al., 2018). However, genetic and molecular mechanisms by which these factors control grain size still remain to be further elucidated.

The ubiquitin proteasome pathway plays a key role in the control of seed size ( $\mathrm{Li}$ and Li, 2016; Li et al., 2019). Many proteins can be ubiquitinated by E3 ubiquitin ligases, and ubiquitinated proteins can also be deubiquitinated by deubiquitinating enzymes. Rice WTG1/OsOTUB1, encoding a deubiquitination enzyme, regulates grain width and thickness by restricting cell expansion in spikelet hulls (Huang et al., 2017; Wang et al., 2017). Rice ubiquitin-specific protease 15 (OsUBP15) positively regulates grain width and grain weight by increasing cell proliferation in spikelet hulls (Shi et al., 2019). The major QTL for grain width and weight (GW2) encodes an E3 ubiquitin ligase in rice (Song et al., 2007). A loss of function mutation of GW2 causes the formation of wide and heavy grains due to the increased cell proliferation in spikelet hulls, resulting in high grain yield. Arabidopsis DA2 shares significant similarity with rice GW2 (Xia et al., 2013). Loss of function of DA2 results in large seeds and organs, suggesting a conserved function between GW2 and DA2 in seed size control. DA2 has been known to interact with the ubiquitin receptor DA1 to control seed size in Arabidopsis (Xia et al., 2013). A recent study has revealed that DA2 can multiply monoubiquitinate DA1 to activate its peptidase activity, thereby causing the cleavage of DA1 substrates (Dong et al., 2017).

Glutaredoxins are glutathione-dependent oxidoreductases that have an important function in oxidative stress responses. Arabidopsis ROXY1/2, the CC-type GRXs, regulate anther and petal development and pathogen response (Wang et al., 2009; Xing and Zachgo, 2008). Overexpression of rice homologs OsROXY1 and OsROXY2 can rescue the flower phenotype of roxyl mutant (Wang et al., 2009). Arabidopsis ROXY1/2 and ROXY19 can interact with several bZIP transcription 
factors PERIANTHIA (PAN) and TGA2/5/6/10 to regulate the flower development and stress responses ( $\mathrm{Li}$ et al., 2009; Murmu et al., 2010; Ndamukong et al., 2007; Zander et al., 2012). It has been proposed that ROXY1/2 may influence the redox state of these bZIP transcription factors, although there is no direct evidence to support this (Li et al., 2009; Murmu et al., 2010). Although GRXs and bZIP transcription factors play crucial roles in flower development, stress responses and nitrogen utilization (Chuang et al., 1999; El-Kereamy et al., 2015; Hong et al., 2012; Kesarwani et al., 2007; Li et al., 2009; Murmu et al., 2010; Running and Meyerowitz, 1996; Wang et al., 2009; Wu et al., 2017; Xu et al., 2019; Zander et al., 2012), their function in seed size control has not been studied in detail. Here we found that $W G 1$ encodes a CC-type glutaredoxin (OsGRX8) and positively regulates grain width in rice. GW2 ubiquitinates WG1 and modulates its stability. WG1 associates with OsbZIP47 and represses its transcriptional activity by associating with the transcriptional co-repressor ASP1. Thus, our findings define a novel genetic and molecular mechanism by which the GW2-WG1-OsbZIP47 regulatory module controls grain size in rice, suggesting that this pathway is crucial for grain size improvement in crops.

\section{Results}

\section{WG1/OsGRX8 positively regulates grain width and weight}

To identify grain size regulators, we sought to overexpress rice genes that are expressed in developing panicles in a japonica variety Zhonghuajing (ZHJ) background (See Materials and Methods). One line carrying the LOC_Os02g30850 gene showed wide, slightly short and heavy grains (Fig. 1A, E-G). We named this gene as WIDE GRAIN $1(W G l)$ and also named this transgenic line as WG1-overexpresion line ( $W G 1-O E$ ). The $W G 1-O E$ also exhibited slightly short plants and panicles (SI Appendix, Fig. S1A-C). To confirm that LOC_Os02g30850 is responsible for wide and heavy grain phenotypes, we re-cloned LOC_Os02g30850 to the same vector with the constitutive expression Actin 1 promoter (McElroy et al., 1990), which was used in WG1-OE, and generated multiple transgenic plants. 
Transgenic plants (proActin:WG1) showed wide, slightly short and heavy grains as well as the reduced plant height and panicle length (Fig. 1A, E-G and SI Appendix, Fig. S1A-E), further supporting that the phenotypes of WG1-OE were caused by overexpression of LOC_Os02g30850.

WG1 encodes a CC-type glutaredoxin (OsGRX8) with catalytic active site CCMC, glutathione binding site Glycine, L**LL motif and ALWL motif (Fig. 1D). Although a function of WG1/OsGRX8 in stress responses has been reported (Sharma et al., 2013), the role of WG1/OsGRX8 in grain size control has not been studied in detail. Homologs of WG1 are found in Arabidopsis, rice and other crop plants (SI Appendix, Fig. S2, 3). WG1 shares high similarity with Arabidopsis ROXY1/2, which regulate anther and petal development (Wang et al., 2009; Xing and Zachgo, 2008).

To further understand the function of WG1 in grain size control, we designed two target sites in the coding region of WG1 using CRISPR/Cas9 technology and generated three loss-of-function mutant alleles wgl-1, wgl-2 and wg1-3. All these mutant lines had base pair(s) deletion in the coding region of $W G 1$, and resulted in reading frame shift (Fig. 1B, C and SI Appendix, Fig. S4A, B). As shown in Fig. 1B, H-J and SI Appendix, Fig. S4C, D, wgl-1, wgl-2 and wgl-3 mutants showed narrower and lighter grains and slightly higher plants than the wild type (ZHJ). By contrast, length of $w g l$ panicles was similar to that of ZHJ panicles. These results show that $W G 1$ regulates grain width and weight as well as organ growth in rice.

$W G 1 / O s G R X 8$ is required for and promotes cell proliferation in the grain-width direction

The size of a grain is coordinately regulated by cell proliferation and cell expansion. The spikelet hull restricts grain growth, therefore determining the final size of a grain in rice ( $\mathrm{Li}$ and $\mathrm{Li}, 2016$; $\mathrm{Li}$ et al., 2019). To understand how WG1 controls grain width, we examined cell number and cell size in the outer lemma of spikelet hulls. As shown in Fig. 1K and SI Appendix, Fig. S4E, cell width in proActin:WG1\#1 and wg1-1 
spikelet hulls was comparable with that in ZHJ spikelet hulls. By contrast, the cell number in the grain-width direction in proActin:WG1\#1 spikelet hulls was significantly increased compared with that in ZHJ spikelet hulls, while the cell number in the grain-width direction in wgl-1 spikelet hulls was significantly decreased compared with that in ZHJ spikelet hulls (Fig. 1L). Therefore, these results indicate that WG1 controls grain width by promoting cell proliferation in the grain-width direction. We also investigated that cell number and cell length in the grain-length direction in proActin:WG1\#1 and wgl-1 spikelet hulls. Both cell number and cell length were decreased slightly but not significantly compared with those in ZHJ spikelet hulls (SI Appendix, Fig. S4F-G). It is a possible reason that proActin:WG1\#1 grains were very slightly shorter than wild-type grains. By contrast, cell number in the grain-length direction in wgl-1 spikelet hulls was significantly increased compared with that in ZHJ spikelet hulls, while cell length in the grain-length direction in wgl-1 spikelet hulls was decreased (SI Appendix, Fig. S4F-G), indicating that increased cell number in the grain-length direction results in the long grain phenotype of $w g 1-1$. These results also suggest a possible compensation mechanism between the grain-width direction growth and the grain-length direction growth.

\section{Expression and subcellular localization of WG1}

To examine tissue-specific expression patterns of $W G 1$, we generated the $W G 1$ promoter:WG1-GUS fusion (proWG1:WG1-GUS) transgenic rice plants. GUS ( $\beta$-glucuronidase) activity in proWG1:WG1-GUS was detected in roots, shoots, coleoptile, and the early stages of developing panicles and grains. Expression of WG1 was decreased at the late stages of panicle and grain development (Fig. 2A). Consistent with this, we tested the transcripts of WGl in developing panicles of the wild type using quantitative real-time RT-PCR analysis and found that relative expression of $W G 1$ was gradually decreased during panicle development (Fig. 2B). 
transgenic lines in ZHJ background. GFP fluorescence in proWG1:WG1-GFP transgenic rice was observed in nuclei (Fig. 2C), indicating that WG1 is nuclear-localized protein. To confirm whether WG1-GFP fusion protein is functional, we crossed proWG1:WG1-GFP with wgl-1 mutant and generated proWG1:WG1-GFP;wg1-1 plants. The proWG1:WG1-GFP;wg1-1 grains were significantly wider than wgl-1 grains (Fig. 2D), indicating that WG1-GFP fusion protein is functional.

\section{GW2 physically interacts with WG1}

To understand how WG1 regulates grain size, we conducted a yeast two-hybrid screen to identify putative WG1-interacting proteins using the cDNA library of young panicles. WG1 was fused to the GAL4 DNA binding domain and used as the bait. Among several interacting proteins, two different clones corresponding to GW2 were identified in this screen. Considering that loss-of-function of GW2 produced wide and heavy grains (Song et al., 2007), like that observed in WG1-OE, it is possible that GW2 interacts with WG1 to control grain size. We then tested whether WG1 could interact with the full length GW2 in yeasts. As shown in Fig. 3A, WG1 interacted with the full length GW2 in yeast cells. The N-terminus of GW2 contains a RING domain, which has E3 ubiquitin ligase activity (Song et al., 2007). To test which regions of GW2 are responsible for the WG1 interaction, we generated two truncated forms of GW2 for yeast two-hybrid assay. GW2 (1-114) contains the RING domain, and GW2 (94-425) possesses the C-terminal region. As shown in Fig. 3A, GW2 (94-425) interacted with WG1, indicating that the C-terminal region of GW2 is required for the interaction between WG1 and GW2.

We then tested whether GW2 could interact with WG1 using split luciferase complementation assays. We fused GW2 to the N-terminus of luciferase to generate GW2-nLUC, and ligated WG1 to the C-terminus of luciferase to generate cLUC-WG1. GW2-nLUC was co-transformed with cLUC-WG1 into N. benthamiana leaves. As shown in Fig. 3B, luciferase activity was observed when we coexpressed 
GW2-nLUC with cLUC-WG1, whereas the negative control did not show luciferase activity, indicating that GW2 associates with WG1 in vivo.

We further asked whether GW2 could associate with WG1 in rice plants. We generated rice transgenic lines expressing pro35S:Myc-GW2 and crossed them with pro35S:GFP or proWG1:WG1-GFP transgenic lines to obtain pro35S:GFP;pro35S:Myc-GW2 and proWG1:WG1-GFP;pro35S:Myc-GW2 plants, respectively. Total proteins from shoots of 10-day old seedlings were isolated and incubated with GFP-Trap-A agarose beads to immunoprecipitate GFP or WG1-GFP complex, respectively. As shown in Fig. 3C, Myc-GW2 was detected in the immunoprecipitated WG1-GFP complex but not in the negative control (GFP), indicating that GW2 physically associates with WG1 in rice plants.

A previous study showed that GFP signal was observed in the cytoplasm by transiently expressing GFP-GW2 in onion epidermal cells (Song et al., 2007). By contrast, WG1 is localized in nuclei. To investigate whether GW2 and WG1 could have the overlapped subcellular localization, we transiently expressed pro35S:GFP-GW2 in N.benthamiana leaves. GFP signals were observed in both the cytoplasm and the nucleus (Fig. S5A). We then isolated the cytoplasmic protein fraction and the nuclear protein fraction using pro35S:Myc-GW2 transgenic plants. Myc-GW2 proteins were detected in both the cytoplasmic protein fraction and the nuclear protein fraction (Fig. 3D). These results showed that GW2 is localized in both the cytoplasm and the nucleus, and also supported that GW2 and WG1 have the overlapped subcellular localization in nucleus. We further asked whether GW2 could interact with WG1 in nucleus. To test this, we adopted the bimolecular fluorescence complementation assays to investigate the interaction between GW2 and WG1. We transiently coexpressed cYFP-GW2 or cYFP (C-terminal fragment of YFP) with nYFP-WG1 or nYFP (N-terminal fragment of YFP) in $N$. benthamiana leaves, respectively. As shown in SI Appendix, Fig. S5, coexpression of cYFP-GW2 with nYFP-WG1 resulted in strong yellow fluorescent protein (YFP) fluorescence in nuclei 
245 of epidermal cells, whereas no YFP fluorescence was observed in the negative controls (nYFP/cYFP) (SI Appendix, Fig. S5B). These results further support that

247 GW2 interacts with WG1 in nucleus.

\section{GW2 ubiquitinates WG1 and modulates its stability}

As GW2 is a functional E3 ubiquitin ligase (Song et al., 2007), we asked whether GW2 could ubiquitinate WG1 protein. We expressed GW2 in Escherichia coli as a fusion protein with maltose binding protein (MBP-GW2). WG1 was expressed as the fusion protein with FLAG (FLAG-WG1) in Escherichia coli. In the presence of an E1 ubiquitin activating enzyme, an E2-conjugating enzyme, HIS-ubiquitin, MBP-GW2 and FLAG-WG1, we detected the ubiquitinated FLAG-WG1 (Fig. 4A). However, in the absence of HIS-ubiquitin or MBP-GW2, no polyubiquitination was detected. These ubiquitination assays indicate that GW2 can ubiquitinate WG1 in vitro.

Considering that GW2 can ubiquitinate WG1, we investigated whether GW2 could influence the degradation of WG1 protein. We examined the protein level of WG1 in plants overexpressing GW2. We measured the protein level of WG1-GFP in proWG1:WG1-GFP;pro35S:Myc-GW2 and proWG1:WG1-GFP plants, respectively. As shown in Fig. 4B and SI Appendix, Fig. S6A-C, the protein levels of WG1-GFP were obviously decreased in pro35S:Myc-GW2 background compared with those in wild-type background, while the transcription level of WG1-GFP was not decreased by overexpression of GW2 (SI Appendix, Fig. S7A). These results indicate that GW2 promotes the degradation of WG1 protein in rice.

We then asked whether that loss of function of GW2 could cause the accumulation of WG1 protein in rice. We generated a loss-of-function mutant gw2-cl (Fig. 4D) by CRISPR/Cas9 technology in ZHJ background. The $g w 2-c 1$ had a 1-bp insertion in the first exon of $G W 2$, resulting in a reading frame shift and a premature stop codon (Fig. 4D). The $g w 2-c 1$ grains were significantly wider than ZHJ grains, supporting that GW2 negatively regulates grain width (Song et al., 2007). We then crossed 
proWG1:WG1-GFP transgenic lines with gw2-cl and obtained

273 proWG1:WG1-GFP;gw2-c1 plants. As shown in Fig. 4C and SI Appendix, Fig. S6D-F, the protein levels of WG1-GFP in $g w 2-c 1$ mutant background were obviously higher than those in wild-type background, whereas the transcription level of WG1-GFP was not increased in the $g w 2-c 1$ mutant background (SI Appendix, Fig. S7B). These results further support that GW2 regulates WG1 protein stability in rice.

\section{GW2 acts in a common pathway with WG1 to control grain width}

As GW2 physically interacts with WG1 protein and modulates its stability, we sought to establish genetic relationships between $G W 2$ and $W G 1$ in grain width control. The wg 1-2 mutant produced narrower grains than the wild type ZHJ (Fig. 4E, F), while the gw2-cl mutant showed much wider grains than ZHJ (Fig. 4E, F). We crossed $g w 2-c 1$ with $w g 1-2$ and generated the $w g 1-2$ gw2-cl double mutant. As shown in Fig. 4E-F and SI Appendix, Fig. S8B, the wg1-2 mutation partially suppressed the wide and heavy grain phenotypes of $g w 2-c 1$, suggesting that $G W 2$ and $W G 1$ may act, at least in part, in a common pathway to control grain width and weight. Similar genetic analyses have been used to explain partial suppression in several studies (Liu et al., 2019; Pham et al., 2018; Wang et al., 2016; Zhai et al., 2015). Considering that both gw2-cl and wg1-2 have longer grains, the wg1-2 mutation enhanced the grain length phenotype of gw2-cl (SI Appendix, Fig. S8A), suggesting that GW2 and WG1 might have different mechanisms to control grain length.

\section{WG1 interacts with OsbZIP47}

In the yeast two-hybrid screen for WG1-interacting proteins, OsbZIP47 was identified as another interacting protein. OsbZIP47 encodes a TGA transcription factor (Nijhawan et al., 2008), and its function in plant growth and development has not been studied yet. OsbZIP47 and its homologs were found in different plant species, such as Oryza sativa, Arabidopsis thaliana, Zea mays, Sorghum bicolor, Setaria italica, Brassica napus, Gossypium hirsutum, Triticum aestivum, Medicago truncatula and Glycine max (SI Appendix, Fig. S9). OsbZIP47 shares similarity with Arabidopsis 
PERIANTHIA (PAN) and TGA transcription factors (SI Appendix, Fig. S10), which is involved in flower development and stress responses (Chuang et al., 1999; Kesarwani et al., 2007; Li et al., 2009; Murmu et al., 2010; Running and Meyerowitz, 1996; Zander et al., 2012). We further confirmed that WG1 interacted with the full length OsbZIP47 in yeast cells (Fig. 5A). To analyze which domain of OsbZIP47 is responsible for the interaction with WG1, we constructed two truncated versions of OsbZIP47 in the yeast two-hybrid assays. OsbZIP47 (1-161) includes the N-terminal bZIP domain, and OsbZIP47 (139-385) contains the C-terminal region. Yeast two hybrid assays showed that the C-terminal region of OsbZIP47 is required for the interaction with WG1 (Fig. 5A).

We then tested whether OsbZIP47 could interact with WG1 using split luciferase complementation assays. We fused WG1 to the N-terminus of luciferase to generate WG1-nLUC. We also fused OsbZIP47 to the C-terminus of luciferase to generate cLUC-OsbZIP47. cLUC-OsbZIP47 was cotransformed with WG1-nLUC into $N$. benthamiana leaves. Luciferase activity was detected when we coexpressed cLUC-OsbZIP47 with WG1-nLUC, whereas the negative controls did not have luciferase activity (Fig. 5B), indicating that WG1 associate with OsbZIP47 in vivo.

To confirm the interaction of OsbZIP47 with WG1 in rice plants, we generated rice transgenic lines expressing pro35S:Myc-OsbZIP47 and crossed it with pro35S:GFP, proWG1:WG1-GFP transgenic lines to obtain pro35S:GFP;pro35S:Myc-OsbZIP47 and proWG1:WG1-GFP;pro35S:Myc-OsbZIP47 plants, respectively. Total proteins of young panicles were isolated and incubated with GFP-Trap-A agarose beads to immunoprecipitate GFP and WG1-GFP complex, respectively. As shown in Fig. 5C, Myc-OsbZIP47 was detected in the immunoprecipitated WG1-GFP complex but not in the negative control (GFP), indicating that WG1 physically associates with OsbZIP47 in rice. 


\section{with the transcriptional co-repressor ASP1}

OsbZIP47 is a TGA transcription factor with a bZIP (Basic Leucine Zipper) domain in the $\mathrm{N}$ terminus (Fig. 5A). To confirm whether OsbZIP47 has transcription activation activity, we adopted dual-luciferase reporter (DLR) assay system in rice protoplasts (Hao et al., 2011). The coding sequence of OsbZIP47 was fused to the DNA sequence encoding the GAL4 DNA-binding domain under the control of $35 S$ promoter and a translational enhancer $\Omega$ to generate the BD-OsbZIP47 effector plasmid (Hao et al., 2011). The firefly luciferase gene (LUC) driven by a mini-35S (TATA box) promoter with five copies of the GAL4 binding element was used as a reporter, and the Renilla luciferase gene driven by the Ubiquitin3 promoter was used as an internal control (Hao et al., 2011). As shown in Fig. 5D, we observed that OsbZIP47 activated the reporter gene, indicating that OsbZIP47 has the transcriptional activation activity. By contrast, coexpression of WG1 decreased the transcriptional activation activity of OsbZIP47 in the protoplasts, indicating that WG1 can repress the transcriptional activation activity of OsbZIP47.

GRXs have been known to modulate target protein activities by S-deglutathionylation reactions or by the reduction of protein disulfide bonds (Stroher and Millar, 2012). WG1 shares similarities with Arabidopsis ROXY1/2 proteins, which influence the functions of the bZIP transcription factors TGAs. However, it is unclear whether ROXYs can modify the redox state of TGA proteins, although indirect evidence showed that the mutation in cysteine (C340S) of PAN, a TGA transcription factor, can not complement the phenotypes of pan mutant ( $\mathrm{Li}$ et al., 2009). Glutathionylation assay showed that OsbZIP47 can be glutathionylated and WG1 can reduce the glutathionylation of OsbZIP47 in vitro (SI Appendix, Fig. S11). To further verify whether WG1-OsbZIP47 could regulate grain size through redox state, we conducted dual-luciferase reporter (DLR) assay in rice ZHJ protoplasts. OsbZIP47 only contains three cysteines (C17, C197 and C269) (SI Appendix, Fig. S12A). We mutated all cysteines in OsbZIP47 into serines (C17S, C197S and C269S). As shown in SI Appendix, Fig. S12B, we found that the transcriptional activation activity of 
OsbZIP47(C17/197/269S) was similar to that of OsbZIP47, suggesting that the deglutathionylation of OsbZIP47 by WG1 might not affect its transcriptional activation activity.

TOPLESS (TPL) is widely recognized as the transcriptional co-repressor (Causier et al., 2012; Ke et al., 2015). TPL has been reported to interact with transcription factors and transcriptional cofactors to influence their transcriptional activity and repress gene expression (Causier et al., 2012; Ke et al., 2015; Pauwels et al., 2010; Uhrig et al., 2017). In Arabidopsis, TPL has been shown to interact with CC-type glutaredoxins that share similarity with WG1 and repress transcriptional activity of TGA-dependent target promoters (Uhrig et al., 2017). We therefore asked whether WG1 could interact with rice TPL and repress the transcriptional activity of OsbZIP47. In rice, aberrant spikelet and panicle1 (ASP1) encodes a TOPLESS-related protein and regulates spikelet, panicle and tiller bud development (Li et al., 2020; Xia et al., 2020; Yoshida et al., 2012). As shown in SI Appendix, Fig. S13A, WG1 interacted with ASP1 in yeast cells. However, GW2 and OsbZIP47 did not interact with ASP1 in yeast cells. The split luciferase complementation assays revealed that WG1 associates with ASP1 in vivo (SI Appendix, Fig. S13B). To further verify whether WG1 interact with ASP1, we performed co-immunoprecipitation analyses. We transiently co-expressed pro35S:GFP-ASP1 with pro35S:Myc-WG1 in N. benthamiana leaves. Co-immunoprecipitation assays showed that WG1 physically associates with ASP1 (SI Appendix, Fig. S13C).

It has been reported that the conserved ALWL motif is indispensable for interaction between CC-type glutaredoxin proteins and TPL/TPL-RELATED2 (TPR2) proteins in Arabidopsis (Uhrig et al., 2017). We then asked whether the ALWL motif in the WG1 is required for the interaction with ASP1. We constructed mutant versions with deletion and mutation in the ALWL motif of WG1 for yeast two-hybrid assays (SI Appendix, Fig. S14A). As shown in SI Appendix, Fig. S14A, WG1 with the deletion or mutation in the ALWL motif did not interact with ASP1. By contrast, the ALWL motif 
400

401

402

403

of WG1 is not required for the interaction with OsbZIP47 (SI Appendix, Fig. S14A). Interestingly, WG1 without the ALWL motif lost the ability to repress the transcriptional activation activity of OsbZIP47 (Fig. 5D). It is therefore possible that WG1 may recruit the transcriptional co-repressor ASP1 through its ALWL motif to repress the transcriptional activation activity of OsbZIP47.

To investigate the function of ASP1 in grain size control, we isolated a mutant asp1-101 from the $\gamma$-ray irradiated M2 populations of the japonica variety Kuanyejing (KYJ). The asp1-101 had a 2-bp deletion in the 24th exon, resulting in a reading frame shift (SI Appendix, Fig. S14B). We examined grains of asp1-101 and found that asp1-101 had elongated glume compared with the wild type KYJ, consistent with a previous study (Yoshida et al., 2012). The asp1-101 grains were also significantly thinner, shorter and lighter than the wild type KYJ (SI Appendix, Fig. S14C-F), indicating that ASP1 influences grain size.

\section{WG1 acts in a common pathway with OsbZIP47 to regulate grain width and} weight

To determine the function of OsbZIP47 in grain size control, we generated the loss-of-function mutant osbzip47-cl using the CRISPR/Cas9 technology in the ZHJ background. The osbzip47-cl had a 1-bp insertion in the 6th exon of OsbZIP47, resulting in a reading frame shift and a premature stop (Fig. 6A and SI Appendix, Fig. S15A). As shown in Fig. 6B and 6E, grains of osbzip47-cl were significantly wider than those of ZHJ, while the grain length, plant height and panicle length of osbzip47-cl was not significantly changed compared with that of ZHJ (Fig. 6F and Fig. S15B, C). The 1,000-grain weight of osbzip47-cl was significantly increased compared with that of ZHJ (Fig. 6G). To verify the grain size phenotype of osbzip47-cl, we introduced OsbZIP47-GFP under its own promoter (proOsbZIP47:OsbZIP47-GFP) to osbzip47-c1. Transgenic lines showed a complementation of the osbzip47-cl grain width phenotype (SI Appendix, Fig. S15D-E). We further expressed the OsbZIP47 gene driven by an Actin promoter in 
ZHJ background and generated transgenic lines. The proActin:OsbZIP47 transgenic plants produced narrower, slightly longer and lighter grains than ZHJ, like those observed in WG1 mutants (Fig. 6C, H-J). The relative expression levels of OsbZIP47 in proActin:OsbZIP47 transgenic lines were correlated with their grain width phenotype (Fig. 6K). Further cellular analysis showed that OsbZIP47 restricts grain growth by decreasing cell proliferation (Fig. S15F-G).

Overexpression of OsbZIP47 causes similar narrow grain phenotype to WG1 mutants, suggesting that WG1 and OsbZIP47 could function in a common pathway to control grain width. To further understand the genetic relationship between WG1 and OsbZIP47, we used the CRISPR/Cas9 technology to knockout OsbZIP47 in wg1-1 mutant background and generated wg1-1 osbzip47-c2 double mutant. The osbzip47-c2 had the same 1-bp insertion in OsbZIP47 as osbzip47-cl (Fig. 6A). The wg1-1 osbzip47-c2 double mutant produced significantly wider and heavier grains than wg 1-1 mutant (Fig. 6D, L-M), indicating that the osbzip47-c2 mutation suppressed the grain size and weight phenotypes of $w g 1-1$. These genetic analyses suggest that WG1 and OsbZIP47 function antagonistically in a common pathway to control grain width and weight in rice.

\section{OsbZIP47 and WG1 have similar expression pattern and subcellular localization} and possess co-regulated genes

We then investigated the expression pattern of OsbZIP47. OsbZIP47 promoter:OsbZIP47-GUS fusion (proOsbZIP47:OsbZIP47-GUS) transgenic rice plants in ZHJ were generated. GUS activity was detected in roots of young seedlings and the early stages of developing panicles and grains (Fig. 6N). By contrast, expression of OsbZIP47 was gradually decreased at the late stages of panicle and grain development (Fig. 6N). We also examined the transcripts of OsbZIP47 in developing panicles of wild type using quantitative real-time RT-PCR analysis and found that relative expression of OsbZIP47 was gradually reduced during panicle development (Fig. 60). The expression patterns of OsbZIP47 were similar to those of 
Considering that OsbZIP47 is the transcription factor, we asked whether OsbZIP47 is localized in the nuclei. We introduced proOsbZIP47:OsbZIP47-GFP to ZHJ. GFP fluorescence in proOsbZIP47:OsbZIP47-GFP was observed to localize in nuclei (Fig. $6 \mathrm{P})$, consistent with its role in transcriptional regulation.

As WG1 and OsbZIP47 act antagonistically in a common pathway to control grain size and have similar expression pattern and subcellular localization, WG1 and OsbZIP47 could possess co-regulated genes. We therefore performed RNA-sequencing (RNA-seq) analysis with ZHJ, proActin:WG1\#1, and osbzip47-c1 young panicles. RNA-seq data showed that $39.4 \%$ of up-regulated genes in osbzip47-c1 exhibited the increased expression in proActin:WG1\#1 (SI Appendix, Fig. S16A). Similarly, $26.9 \%$ of down-regulated genes in osbzip47-c1 showed the decreased expression in proActin:WG1\#1 (SI Appendix, Fig. S16B). Gene Ontology (GO) assay indicated that WG1 and OsbZIP47-regulated genes were enriched to similar GO terms (SI Appendix, Fig. S17-19). These results further support that WG1 and OsbZIP47 have the overlapped function. Expression of genes involved in transcriptional regulation, hormone signaling, carbohydrate metabolic process, and cell cycle were regulated by WG1 and OsbZIP47 (SI Appendix, Spreadsheet S1), suggesting that WG1 and OsbZIP47 may control grain growth by influencing multiple biological processes.

\section{DISSCUSSION}

Regulation of seed size is a fundamental question in developmental biology, but the knowledge on how plants determine their seed size is limited despite its central importance. Here we identify the WG1 as positive regulators of grain size and weight. WG1 controls grain width and weight by promoting cell proliferation in spikelet hulls. Consistent with its function in cell proliferation, expression of WG1 was higher in young panicles than that in older ones. WG1 encodes CC-type glutaredoxin protein 
471 OsGRX8. Homologs of WG1 in plant species have been described to affect stress 472 responses, nitrogen utilization, flower development and seed development 473 (El-Kereamy et al., 2015; Hong et al., 2012; Li et al., 2009; Wang et al., 2009; Wu et 474 al., 2017; Xu et al., 2019; Zander et al., 2012), but how WG1 and its homologs in 475 plants regulate seed size has not been studied in detail. It is also a big challenge to 476 investigate whether WG1 could coordinate stress responses, nitrogen utilization and 477 grain growth in the future.

To understand the mechanism of WG1 in grain size control, we identified several WG1-interacting proteins. One of them is a functional E3 ubiquitin ligase GW2, which has been known to control grain width and weight by restricting cell proliferation in the spikelet hulls (Song et al., 2007). Loss of function of GW2 results in wide and heavy grains, like those observed in plants overexpressing WG1. GW2 is recognized as a crucial regulator of grain size because GW2 and its homologs in Arabidopsis, wheat and maize share conserved function in seed size control ( $\mathrm{Li}$ and $\mathrm{Li}$, 2016; Li et al., 2019). However, the substrates of GW2 in grain size control have not been identified so far. In this study, we reveal that WG1 is a direct substrate of GW2 for protein degradation. Supporting this, we demonstrate that GW2 can ubiquitinate WG1 and modulates its stability. We further show that GW2 and WG1 have an overlapping subcellular localization in nucleus. Consistent with biochemical data, genetic analyses suggest that GW2 and WG1 function, at least in part, in a common pathway to control grain width. Considering that $w g$ 1-2 partially represses the grain width phenotype of $g w 2-c 1$, it is possible that GW2 could have other substrates for protein degradation in grain size control. GW2 shares high similarity with Arabidopsis DA2, which has been reported to multiply monoubiquitinate the peptidase DA1 to control seed size in Arabidopsis (Dong et al., 2017). It is a worthwhile challenge to investigate whether rice DA1 could affect grain size and GW2 could monoubiquitinate rice DA1 in the future. 
transcription factor OsbZIP47 as another WG1-interacting protein. Homologs of OsbZIP47 in plant species have been reported to influence stress responses and flower development (Chuang et al., 1999; Kesarwani et al., 2007; Li et al., 2009; Murmu et al., 2010; Running and Meyerowitz, 1996; Zander et al., 2012), but the function of OsbZIP47 and its homologs in seed size control is totally unknown. Here we reveal that OsbZIP47 is a negative regulator of grain width and weight. Genetic analyses suggest that WG1 acts with OsbZIP47 in a common genetic pathway to control grain width and weight in rice. Supporting this result, OsbZIP47 shows similar expression pattern and subcellular localization to WG1 in rice. RNA-seq data also showed that WG1 and OsbZIP47 have the overlapped function in regulating gene expression. WG1 represses the transcriptional activity of OsbZIP47. However, deglutathionylation of OsbZIP47 by WG1 might not affect its transcriptional activation activity. Interestingly, we found that WG1 interacts with the transcriptional co-repressor ASP1 that is a TOPLESS-related protein (Yoshida et al., 2012). TOPLESS proteins have been recognized as general co-repressors that are recruited to different pathways by specific adaptor proteins in plants (Causier et al., 2012; Ke et al., 2015; Ma et al., 2017; Pauwels et al., 2010; Uhrig et al., 2017). It is plausible that WG1 may function as an adaptor protein. The interaction between WG1 and ASP1 depends on the ALWL motif of WG1. Importantly, the deletion of ALWL motif abolished the repression effect of WG1 on the transcriptional activity of OsbZIP47. It is an accepted concept that the transcriptional co-repressors usually directly or indirectly associate with the transcription factors to repress their transcriptional activity and influence expression of downstream genes. Thus, it is possible that WG1 may act as adaptor proteins and repress the transcriptional activity of OsbZIP47 by recruiting the transcriptional co-repressor ASP1. Supporting this idea, Arabidopsis homologs of WG1 have been shown to recruit the transcriptional co-repressor TOPLESS to repress the activation of TGA-dependent target promoters (Uhrig et al., 2017).

Based on these molecular and genetic evidences, we proposed a working model for 
GW2-WG1-OsbZIP47-mediated control of grain size in rice. The growth signals may activate WG1 to repress the transcriptional activation activity of OsbZIP47 by recruiting the transcriptional co-repressor ASP1 (Fig. 7). This may repress expression of OsbZIP47-targeted genes promoting cell proliferation. When the growth signals become weaker or disappear, the E3 ubiquitin ligase GW2 can ubiquitinate WG1 and cause the degradation of WG1, which releases the repressive effect of WG1-ASP1 on OsbZIP47 transcriptional activation activity. This promotes expression of OsbZIP47-targeted genes involved in transcriptional regulation, hormone signaling, carbohydrate metabolic processes, and cell cycle, thereby limiting grain growth (Fig. 7). Therefore, our findings define a novel genetic and molecular mechanism by which the GW2-WG1-OsbZIP47 regulatory module functions to control grain size and weight in rice. Considering that GW2,WG1 and OsbZIP47 are expressed in both developing grains and vegetative organs, it is possible that GW2-WG1-OsbZIP47 module may also affect vegetative organ development. Supporting this, GW2 regulates rice growth during vegetative and reproductive phases (Shim et al., 2020; Song et al., 2007). Transgenic plants (proActin:WG1) affect grain size as well as plant height. Although osbzip47-cl does not show clear defects in vegetative organ growth, it is also possible that OsbZIP47 may act redundantly with its family members to regulate vegetative growth. Similarly, several key factors of seed size control influence both seed and organ growth in rice and Arabidopsis, such as GS3, GW5, DA1 and DA2 (Li et al., 2008; Liu et al., 2017; Mao et al., 2010; Xia et al., 2013).

Seed size is a key trait for crop breeding. Here we discover that the GW2-WG1-OsbZIP47 pathway controls grain size in rice. GW2, WG1 and OsbZIP47 share similarity with their respective homologs in other crops. For example, homologs of GW2 in wheat has been reported to regulate seed weight (Zhang et al., 2018). It will be a challenge to investigate the functions of the GW2-WG1-OsbZIP47 module in key crops and exploit their homologs and natural alleles to increase grain size and weight in the future. 


\section{Materials and Methods}

\section{Plant materials and growth conditions}

Genes with relatively high expression in young panicles were previously subcloned into the vector pIPKb003(Himmelbach et al., 2007) with the Actin 1 promoter and overexpressed in a japonica variety Zhonghuajing (ZHJ). The highly expressed genes were determined by Affymetrix Rice Genome array and selected according to their predicted biological functions. Overexpression of the LOC_Os02g30850 gene caused wide and heavy grains and named this gene as WIDE GRAIN 1 (WG1) and named this original transgenic line as WG1-overexpresion line (WG1-OE).

The Zhonghuajing (ZHJ), WG1-OE, wg1-1, wg1-2, wg1-3, gw2-c1, osbzip47-c1 and other plants were grown in experimental field in Lingshui (Hainan province, China) from December to April the next year, Changping district in Beijing from May to October or Zhejiang Academy of Agricultural Sciences in Hangzhou from June to November. Mutants of WG1, GW2 and OsbZIP47 were obtained by CRISPR/Cas9 technology. Multiple T1 generation lines of each allelic mutation were selected for self-crossing, and the T2 generation population was obtained, in which transgenes were identified, and non-transgenic lines were selected for the next generation planting. Thereafter, we obtained stable inherited mutants wg1-1, wg1-2, wg1-3, gw2-c1, osbzip47-c1 and osbzip47-c2. All phenotypic experiments were performed on the basis of stable inherited mutant lines without transgene.

\section{Morphological and cellular analysis}

Plant height, panicle length, grain length, grain width and 1,000-grain weight were investigated at maturity stage. Grains from main panicles were collected for grain length and grain width measurement using Scan Maker i560 (Wanshen company, China). For cell size measurement, mature grains were scanned with frozen scanning electron microscope after gold spraying treatment and original figures of $35 \times$ (or $30 \times$, $18 \times$ ) field and $200 \times$ field were saved. The outer epidermal cells in the central part of lemmas (200× field) were measured by ImageJ, and cell number was counted in the 
grain-width direction (the widest part of the grain, $18 \times, 35 \times$ or $30 \times$ field).

589

590

591

592

593

594

595

596

597

598

599

600

601

602

603

604

605

606

607

608

609

610

611

612

613

614

615

\section{RNA isolation, reverse transcription and quantitative Real-time PCR assays} RNA isolation was performed using Tiangen Plant RNA extraction Kit. Reverse transcription was implemented using Invitrogen reverse transcription Kit. Luna Universal qPCR Master Mix (NEB company) was used for quantitative Real-time PCR in the equitment of Bio-rad CFX 96 or Eppendorf Mastercycler ep realplex. Primers used were listed in SI Appendix, Table S1.

\section{Constructs and plant transformation}

All the constructs were generated using the GBclonart Seamless Clone Kit (GB2001-48, Genebank Biosciences). Primers for PCR amplification were listed in SI Appendix, Table S1. We amplified CDSs of WG1 and OsbZIP47 from cDNA of ZHJ, and inserted the fragment to linearized pIPKbO03 vector digested with Sal I and Hind III to generate proActin:WG1 and proActin:OsbZIP47, respectively. For CRISPR/Cas9 construction, we used overlapping PCR method to add the target site sequence to gRNA scaffold. For example, fragment 1 was amplified by F1 and CRI-GW2-R1, fragment 2 was amplified by CRI-GW2-F2 and R2 from a template containing rice gRNA. The two fragments together were reamplified by F1 and R2. Then the products were infused to the linearized pMDC99-cas9 vector digested with Hind III (Feng et al., 2013). The 2,554-bp WG1 genome sequences (containing 2,149-bp fragment upstream of ATG and 405-bp coding region) and 4,672-bp OsbZIP47 genome sequences (containing 2,696-bp fragment upstream of ATG and 1,976-bp coding region) were amplified from DNA of ZHJ and then inserted to linearized pMDC164 (BioVector NTCC Inc., digested with Xba I) and pMDC107 (BioVector NTCC Inc., digested with Asc I and BamH I) vectors to generate proWG1:WG1-GUS, proOsbZIP47:OsbZIP47-GUS, proWG1:WG1-GFP and proOsbZIP47:OsbZIP47-GFP plasmids, respectively. We transferred the plasmids to Agrobacterium strain GV3101 using electroporation method and then transformed them to ZHJ according to the method described previously (Hiei et al., 1994). 
616

617

618

619

620

621

622

623

624

625

626

627

628

629

630

631

632

633

634

635

636

637

638

639

640

641

642

643

\section{GUS staining and detection of GFP fluorescence}

Seedlings and different length of developing panicles of proWG1:WG1-GUS and proOsbZIP47:OsbZIP47-GUS transgenic plants were stained in the same X-gluc buffer solution as described previously (Li et al., 2004). Samples were vacuumed for $1 \mathrm{~h}$ and then incubated in $37^{\circ} \mathrm{C}$ for $6 \mathrm{hrs}$. Finally, chlorophyll was removed by $70 \%$ ethanol, and samples were photographed with the camera of NIKON. GFP fluorescence in roots of 7-day-old seedlings of proWG1:WG1-GFP and proOsbZIP47:OsbZIP47-GFP transgenic plants was observed using NIKON A1 confocal microscopy after treated with nucleus dyer DAPI $\left(1.5 \mu \mathrm{g} \mathrm{ml}^{-1}\right)$ for 20 mins.

The cDNA sequence of GW2 was infused to pMDC43 (BioVector NTCC Inc., digested with Asc I and Sac I) to generate pro35S:GFP-GW2. Primers were listed in SI Appendix, Table S1. Agrobacterium strain GV3101 containing pro35S:GFP-GW2 was transformed to N.benthamiana leaves, respectively. After 48 hrs, GFP fluorescence was observed using Zeiss LSM 710 confocal microscopy after leaves treated with nucleus dyer DAPI $\left(1.5 \mu \mathrm{g} \mathrm{ml}^{-1}\right)$ for 20 mins.

\section{Yeast two-hybrid assay}

We used the Matchmaker Gold Yeast Two-Hybrid system (Clotech) to perform the yeast two-hybrid screen or assay. Young panicles of ZHJ shorter than $5 \mathrm{~cm}$ were used for constructing expression cDNA library for yeast two-hybrid screen. The yeast strain AH109 or GOLD was used for transformation. The CDSs of WG1, WG1 $\triangle$ ALWL, WG1mALWL and ASP1 amplified by primers (SI Appendix, Table S1) were infused to the linearized bait vector $p G B K T 7$ (digested with Nco I and Pst I) to generate $B D-W G 1, B D-W G 1 \triangle A L W L, B D-W G 1 m A L W L$ and $B D-A S P 1$, respectively. The CDSs of GW2 (aa. 1-425), GW2 (aa. 1-114), GW2 (aa. 94-425), OsbZIP47 (aa. 1-385), OsbZIP47 (aa. 1-161), OsbZIP47 (aa. 139-385), WG1 and ASPl were infused to prey vector $p G A D T 7$ (digested with EcoR I and $S a c$ I) to generate $A D-G W 2$, $A D-G W 2(1-114), \quad A D-G W 2(94-425), \quad A D-O s b Z I P 47, \quad A D-O s b Z I P 47(1-161)$, 
$A D$-OsbZIP47(139-385), AD-WG1 and AD-ASP1, respectively.

\section{Bimolecular fluorescence complementation assay}

The CDSs of WG1 and GW2 were cloned into the linearized pMDC43-nYFP and pMDC43-cYFP vectors (digested with Spe I and Sal I) to generate $n Y F P$-WG1 and cYFP-GW2 constructs, respectively. Primers used were listed in SI Appendix, Table S1. Different combinations of Agrobacterium GV3101 containing above plasmids were co-transformed to $N$. benthamiana leaves. After 48 h, YFP fluorescence in leaves was observed using LSM710 confocal laser scanning microscopy (Zeiss).

\section{Protein purification and ubiquitination assay}

The cDNA sequences of WGI and OsbZIP47 were cloned to EcoR I and Xho I sites of the pETNT (Dong et al., 2017) vector to generate FLAG-WG1, WG1-HIS and FLAG-OsbZIP47 constructs, respectively. GW2 was cloned to BamH I site of pMAL-C2 vector (NEB \#E8000S) to generate the MBP-GW2 construct. Primers were listed in SI Appendix, Table S1. Protein purification method was as described previously (Xia et al., 2013). The ubiquitination assay was also conducted according to a previous method (Xia et al., 2013). Polyubiquitinated proteins were detected by immunoblotting with anti-MBP (NEB \#E8032), anti-FLAG (Abmart M20008) and anti-ubiquitin (CST 3936S) antibodies, respectively.

\section{S-glutathionylation assay}

For in vitro glutathionylation assays, $2 \mu \mathrm{g}$ of purified FLAG-OsbZIP47 was incubated with different concentrations of WG1-HIS and initiated by the addition of $5 \mathrm{mM}$ GSSG (Sigma G4501), the mixture were incubated for $10 \mathrm{~min}$ at $30^{\circ} \mathrm{C}$. The half-volume mixture of reactions were stopped by the addition of an equal volume of $2 \times$ non-reduced SDS/PAGE buffer (without $\beta$-mercaptoethanol and DTT) or $2 \times$ reduced SDS/PAGE buffer (with 2\% $\beta$-mercaptoethanol and $200 \mathrm{mM}$ DTT) (Bender et al., 2015). Anti-Glutathione (Abcam ab19534) antibody was used to detect glutathionylation. The remaining mixture of reactions were mixed with an equal 
volume of $2 \times$ reduced SDS/PAGE buffer. Anti-FLAG (Abmart M20008) and anti-HIS (Abmart M20020) antibodies were used to detect FLAG-OsbZIP47 and WG1-HIS as loading control.

\section{In vivo co-immunoprecipitation}

The cDNA sequences of $W G 1, G W 2, O s b Z I P 47$ and $A S P 1$ were infused to $p M D C 43$ (digested with Asc I and Sac I) and pCambial1300-221-Myc(Liu et al., 2011) (digested with BamH I and Pac I) to generate pro35S:GFP-ASP1, pro35S:Myc-WG1, pro35S:Myc-GW2 and pro35S:Myc-OsbZIP47, respectively. Primers for these constructs were listed in SI Appendix, Table S1.

Different combinations of Agrobacterium GV3101 with pro35S:GFP/pro35S:Myc-WG1 and pro35S:GFP-ASP1/pro35S:Myc-WG1 plasmids were co-transformed to $N$. benthamiana leaves. Total proteins were extracted with protein extraction buffer (50mM Tris [pH7.5], 150mM NaCl, 10\% glycerol, $2 \%$ Triton, 1mM EDTA and 1/4 tablet of protease inhibitor cocktail [Roche]/10ml) and incubated with $15 \mu \mathrm{l}$ GFP-Trap-A for $45 \mathrm{~min}$ at $4^{\circ} \mathrm{C}$. Beads were washed for four times with the wash buffer $(50 \mathrm{mM}$ Tris [pH7.5], $150 \mathrm{mM} \mathrm{NaCl}, 10 \%$ glycerol, $0.1 \%$ Triton, $1 \mathrm{mM}$ EDTA and $1 / 4$ tablet of protease inhibitor cocktail [Roche]/10ml). The immunoprecipitates were analyzed by SDS-PAGE and immunoblotted by anti-GFP (Abmart M20004) and anti-Myc (Abmart M20002) antibodies, respectively. The western blot was conducted according to one-step method (Kangwei company, China). Gel image analyzing system Tanon 3500 was used to detect chemiluminescence signals.

Total proteins from proWG1:WG1-GFP;pro35S:Myc-OsbZIP47, pro35S:GFP;pro35S:Myc-OsbZIP47, proWG1:WG1-GFP;pro35S:Myc-GW2 and pro35S:GFP;pro35S:Myc-GW2 plants were extracted and incubated with GFP-Trap-A agarose for $1 \mathrm{~h}$ at $4^{\circ} \mathrm{C}$. Beads were washed for four times with the wash buffer. The immunoprecipitates were analyzed by SDS-PAGE and immunoblotted by 
anti-GFP and anti-Myc antibodies, respectively.

\section{Subcellular fractionation and immunoblot assay}

Young seedlings of pro35S:Myc-GW2 plants were used to isolate the nuclear protein fraction $(\mathrm{N})$ and the cytoplasmic protein fraction $(\mathrm{C})$ according to methods described previously (Huang et al., 2017). Myc-GW2, Luminal-binding protein Bip and Histone H4 were immunoblotted with anti-Myc (Abmart M20002), anti-Bip (Agrisera AS09-481) and anti-H4 (Active Motif) antibodies, respectively.

\section{Rice protoplast preparation and transcriptional activation assay}

The rice protoplast preparation and transformation were performed as a previous report (Zhang et al., 2011). The transcriptional activation activity of OsbZIP47 was conducted using the dual-luciferase reporter (DLR) assay in rice ZHJ protoplasts (Hao et al., 2011). 5×GAL4-LUC was used as reporter. pPTRL was used as internal control. $B D$ was used as negative control. The CDS of OsbZIP47 was fused to the linearized vector $35 S-B D$ (digested with $E c o$ R I and $S a l$ I) to generate $B D$-OsbZIP47. The cDNA sequences of $W G 1$ and $W G 1 \triangle A L W L$ were fused to the linearized vector $p R T 107$ (digested with EcoR I and Sal I) to generate $35 S$-WG1 and $35 S-W G 1 \triangle A L W L$ respectively. For BD-OsbZIP47(C17/197/269S) construction, we used overlapping PCR method to mutate the three cysteines to serines step by step. First, fragment 1 was amplified by GAL4-AD-F and R1, and fragment 2 was amplified by F1 and R from $A D$-OsbZIP47 vector. Fragment 1 and 2 together were reamplified by $\mathrm{F}$ and $\mathrm{R}$. Then the products were infused to the linearized $35 S-B D$ (digested with EcoR I and $S a l$ I) to generate $B D-O s b Z I P 47(C 17 S)$ vector. Second, fragment 3 was amplified by $\mathrm{F}$ and R2, and fragment 4 was amplified by F2 and R from $B D-O \operatorname{sbZIP} 47(C 17 S)$ vector. Fragment 3 and 4 together were reamplified by $\mathrm{F}$ and $\mathrm{R}$. Then the products were infused to the linearized $35 S-B D$ (digested with EcoR I and Sal I) to generate BD-OsbZIP47(C17/197S) vector. At last, fragment 5 was amplified by $\mathrm{F}$ and R3, and fragment 6 was amplified by $\mathrm{F} 3$ and $\mathrm{R}$ from $B D$-OsbZIP47(C17/197S) vector. Fragment 5 and 6 together were reamplified by $\mathrm{F}$ and $\mathrm{R}$. Then the products were 
infused to the linearized $35 S-B D$ (digested with EcoR I and Sal I) to generate BD-OsbZIP47(C17/197/269S) vector. Primers for these constructs were listed in SI Appendix, Table S1. BD-OsbZIP47, BD-OsbZIP47(C17/197/269S), 35S-WG1 and $35 S-W G 1 \triangle A L W L$ were used as effectors. The quantity of effectors, reporter and pPTRL was $8+8 \mu \mathrm{g}, 8 \mu \mathrm{g}$ and $1 \mu \mathrm{g}$ in each protoplast transformation. Protoplasts were incubated for $16 \mathrm{hrs}$ after transformation. Then, the firefly luciferase and Renilla luciferase activity were tested using dual-luciferase reporter (DLR) assay kit in 96 microplate luminometer instrument from Promega.

\section{Firefly luciferase complementation assay}

The cDNA sequences of WGI and OsbZIP47 were fused to the vector pCAMBIA-split_cLUC(Zhou et al., 2018) (digested with BamH I and Sal I) to generate $c L U C-W G 1$ and $c L U C$-OsbZIP47, respectively. The cDNA sequences of $W G 1, G W 2$ and ASP1 were fused to the vector pCAMBIA-split_nLUC(Zhou et al., 2018) (digested with BamH I and Sal I) to generate WG1-nLUC, GW2-nLUC and ASP1-nLUC, respectively. Primers for these constructs were listed in SI Appendix, Table S1. Different combinations of Agrobacterium GV3101 containing above plasmids were co-transformed to $N$. benthamiana leaves. After $48 \mathrm{hrs,} \mathrm{luciferin} \mathrm{was}$ smeared on the leaves to test luciferase intensity in NightOWL II LB983 imaging apparatus (Chen et al., 2008).

\section{RNA sequencing analysis}

The mRNA samples for RNA-seq were extracted from $1 \mathrm{~cm}$ panicles of ZHJ, proActin:WGI\#1 and OsbZIP47-cl with three replicates for each material. Then nine libraries were constructed and sequenced using the Illumina NovaSeq6000 platform (BerryGenomics company). Approximately 20,000,000 clean reads were obtained for each sample and then mapped to the Nipponbare reference genome by Tophat and bowtie2 software. Gene expression level was manifested by fragments per kilobase per million mapped reads (FPKM) value and calculated using RESM software (BerryGenomics company) for each gene. The differential expression analysis was 
performed by edgeR software (BerryGenomics company). Differentially expressed genes were limited to false discovery rate (FDR) $<0.05$ and absolute value of $\log _{2}$ fold change $\geqslant 1$ between proActin:WGI\#1 or OsbZIP47-c1 and ZHJ samples. GO enrichment analysis was accomplished through agriGO v2 program on the 2010 version and MSU7.0 gene ID (TIGR) as reference(Tian et al., 2017).

\section{Acknowledgements}

We would like to thank Prof. Shouyi Chen and Prof. Jinsong Zhang for the dual-luciferase reporter (DLR) vectors and Prof. Jiankang Zhu for the Cas 9 vector. This work is supported by grants from the National Natural Science Foundation of China (3181101602 Y.L; 91735302 Y.L; 3187080209 N.L; 91735304 Q.Q; 31571742 D.W), the strategic priority research program of the Chinese Academy of Sciences (XDB27010102), the National Basic Research Program of China (2018YFD1000706-10 X.R; 2016YFD0100501 N.L; 2016YFD0100402 P.D; 2016YFD0100401 D.W; 2017YFD0101701 X.R), and the National Special Project (2016ZX08009003-003).

\section{Data availability}

The authors declare that the data supporting the findings of this study are available within the Article or are available from the corresponding authors upon reasonable request.

\section{Author contributions}

J.H., D.W., Y.W., R.X., P.D., D.Z., G.D., B.Z., N.L., K.H., L.Z. performed experiments. Y.L conceived this project. Y.L., Q.Q. and I.D. discussed the research plan, and Y.L and J.H designed experiments. J.H., Q.Q., I.D. and Y.L. analyzed data and discussed manuscript. J.H. and Y.L. wrote the article.

\section{Declaration of Interests}

The authors have no competing interests. 


\section{References}

Bender, K.W., Wang, X., Cheng, G.B., Kim, H.S., Zielinski, R.E., and Huber, S.C. (2015). Glutaredoxin AtGRXC2 catalyses inhibitory glutathionylation of Arabidopsis BRI1-associated receptor-like kinase 1 (BAK1) in vitro. The Biochemical journal 467:399-413.

Causier, B., Ashworth, M., Guo, W., and Davies, B. (2012). The TOPLESS Interactome: A Framework for Gene Repression in Arabidopsis. Plant Physiol 158:423-438.

Che, R., Tong, H., Shi, B., Liu, Y., Fang, S., Liu, D., Xiao, Y., Hu, B., Liu, L., Wang, H., et al. (2015). Control of grain size and rice yield by GL2-mediated brassinosteroid responses. Nature plants 2:15195.

Chen, H., Zou, Y., Shang, Y., Lin, H., Wang, Y., Cai, R., Tang, X., and Zhou, J.M. (2008). Firefly luciferase complementation imaging assay for protein-protein interactions in plants. Plant Physiol 146:368-376.

Chuang, C.-F., Running, M.P., Williams, R.W., and Meyerowitz, E.M. (1999). The PERIANTHIA gene encodes a bZIP protein involved in the determination of floral organ number in Arabidopsis thaliana. Genes \& Dev 13:334-344.

Dong, H., Dumenil, J., Lu, F.H., Na, L., Vanhaeren, H., Naumann, C., Klecker, M., Prior, R., Smith, C., McKenzie, N., et al. (2017). Ubiquitylation activates a peptidase that promotes cleavage and destabilization of its activating E3 ligases and diverse growth regulatory proteins to limit cell proliferation in Arabidopsis. Genes \& Dev 31:197-208.

Duan, P., Ni, S., Wang, J., Zhang, B., Xu, R., Wang, Y., Chen, H., Zhu, X., and Li, Y. (2015). Regulation of OsGRF4 by OsmiR396 controls grain size and yield in rice. Nat Plants 2:1-5.

Duan, P., Rao, Y., Zeng, D., Yang, Y., Xu, R., Zhang, B., Dong, G., Qian, Q., and Li, Y. (2014). SMALL GRAIN 1, which encodes a mitogen-activated protein kinase kinase 4, influences grain size in rice. Plant J 77:547-557.

Duan, P., Xu, J., Zeng, D., Zhang, B., Geng, M., Zhang, G., Huang, K., Huang, L., Xu, R., Ge, S., et al. (2017). Natural Variation in the Promoter of GSE5 Contributes to Grain Size Diversity in Rice. Molecular plant 10:685-694.

El-Kereamy, A., Bi, Y.M., Mahmood, K., Ranathunge, K., Yaish, M.W., Nambara, E., and Rothstein, S.J. (2015). Overexpression of the CC-type glutaredoxin, OsGRX6 affects hormone and nitrogen status in rice plants. Frontiers in plant science 6:934.

Fan, C., Xing, Y., Mao, H., Lu, T., Han, B., Xu, C., Li, X., and Zhang, Q. (2006). GS3, a major QTL for grain length and weight and minor QTL for grain width and thickness in rice, encodes a putative transmembrane protein. Theor Appl Genet 112:1164-1171.

Feng, Z., Zhang, B., Ding, W., Liu, X., Yang, D.L., Wei, P., Cao, F., Zhu, S., Zhang, F., Mao, Y., et al. (2013). Efficient genome editing in plants using a CRISPR/Cas system. Cell Res 23:1229-1232.

Guo, T., Chen, K., Dong, N.Q., Shi, C.L., Ye, W.W., Gao, J.P., Shan, J.X., and Lin, 
H.X. (2018). GRAIN SIZE AND NUMBER1 Negatively Regulates the OsMKKK10-OsMKK4-OsMPK6 Cascade to Coordinate the Trade-off between Grain Number per Panicle and Grain Size in Rice. Plant Cell 30:871-888.

Hao, Y.J., Wei, W., Song, Q.X., Chen, H.W., Zhang, Y.Q., Wang, F., Zou, H.F., Lei, G., Tian, A.G., and Zhang, W.K. (2011). Soybean NAC transcription factors promote abiotic stress tolerance and lateral root formation in transgenic plants. Plant $\mathbf{J}$ 68:302-313.

Hiei, Y., Ohta, S., Komari, T., and Kumashiro, T. (1994). Efficient transformation of rice (Oryza sativa L.) mediated by Agrobacterium and sequence analysis of the boundaries of the T - DNA. Plant J 6:271-282.

Himmelbach, A., Zierold, U., Hensel, G., Riechen, J., Douchkov, D., Schweizer, P., and Kumlehn, J. (2007). A set of modular binary vectors for transformation of cereals. Plant Physiol 145:1192-1200.

Hong, L., Tang, D., Zhu, K., Wang, K., Li, M., and Cheng, Z. (2012). Somatic and reproductive cell development in rice anther is regulated by a putative glutaredoxin. Plant Cell 24:577-588.

Hu, J., Wang, Y., Fang, Y., Zeng, L., Xu, J., Yu, H., Shi, Z., Pan, J., Zhang, D., Kang, S., et al. (2015). A Rare Allele of GS2 Enhances Grain Size and Grain Yield in Rice. Molecular plant 8:1455-1465.

Hu, Z., He, H., Zhang, S., Sun, F., Xin, X., Wang, W., Qian, X., Yang, J., and Luo, X. (2012). A Kelch Motif-Containing Serine/Threonine Protein Phosphatase Determines the Large Grain QTL Trait in Rice. Journal of integrative plant biology 54:979-990.

Hu, Z.J., Lu, S.J., Wang, M.J., He, H.H., Sun, L., Wang, H.R., Liu, X.H., Jiang, L., Sun, J.L., Xin, X.Y., et al. (2018). A Novel QTL qTGW3 Encodes the GSK3/SHAGGY-Like Kinase OsGSK5/OsSK41 that Interacts with OsARF4 to Negatively Regulate Grain Size and Weight in Rice. Molecular Plant 11:736-749.

Huang, K., Wang, D., Duan, P., Zhang, B., Xu, R., Li, N., and Li, Y. (2017). WIDE AND THICK GRAIN 1, which encodes an otubain-like protease with deubiquitination activity, influences grain size and shape in rice. Plant $\mathbf{J}$ 91:849-860.

Ke, J., Ma, H., Gu, X., Thelen, A., Brunzelle, J.S., Li, J., Xu, H.E., and Melcher, K. (2015). Structural basis for recognition of diverse transcriptional repressors by the TOPLESS family of corepressors. Sci Adv 1:e1500107.

Kesarwani, M., Yoo, J., and Dong, X. (2007). Genetic interactions of TGA transcription factors in the regulation of pathogenesis-related genes and disease resistance in Arabidopsis. Plant Physiol 144:336-346.

Li, N., and Li, Y. (2016). Signaling pathways of seed size control in plants. Curr Opin Plant Biol 33:23-32.

Li, N., Xu, R., and Li, Y. (2019). Molecular Networks of Seed Size Control in Plants. Annu Rev Plant Biol 70:435-463.

Li, S., Lauri, A., Ziemann, M., Busch, A., Bhave, M., and Zachgo, S. (2009). Nuclear activity of ROXY1, a glutaredoxin interacting with TGA factors, is required for petal development in Arabidopsis thaliana. Plant Cell 21:429-441. 
Li, Y.-F., Zeng, X.-Q., Li, Y., Wang, L., Zhuang, H., Wang, Y., Tang, J., Wang, H.-L., Xiong, M., Yang, F.-Y., et al. (2020). MULTI-FLORET SPIKELET 2, a MYB Transcription Factor, Determines Spikelet Meristem Fate and Floral Organ Identity in Rice. Plant Physiol 184:988-1003.

Li, Y., Sorefan, K., Hemmann, G., and Bevan, M.W. (2004). Arabidopsis NAP and PIR regulate actin-based cell morphogenesis and multiple developmental processes. Plant physiology 136:3616-3627.

Li, Y., Zheng, L., Corke, F., Smith, C., and Bevan, M.W. (2008). Control of final seed and organ size by the DA1 gene family in Arabidopsis thaliana. Genes \& Dev 22:1331-1336.

Liu, J., Chen, J., Zheng, X., Wu, F., Lin, Q., Heng, Y., Tian, P., Cheng, Z., Yu, X., Zhou, K., et al. (2017). GW5 acts in the brassinosteroid signalling pathway to regulate grain width and weight in rice. Nat Plants 3:1-7.

Liu, L., Cui, F., Li, Q., Yin, B., Zhang, H., Lin, B., Wu, Y., Xia, R., Tang, S., and Xie, Q. (2011). The endoplasmic reticulum-associated degradation is necessary for plant salt tolerance. Cell Res 21:957-969.

Liu, Q., Han, R., Wu, K., Zhang, J., Ye, Y., Wang, S., Chen, J., Pan, Y., Li, Q., Xu, X., et al. (2018). G-protein betagamma subunits determine grain size through interaction with MADS-domain transcription factors in rice. Nature communications 9:852.

Liu, Z., Chen, G., Gao, F., Xu, R., Li, N., Zhang, Y., and Li, Y. (2019). Transcriptional Repression of the APC/C Activator Genes CCS52A1/A2 by the Mediator Complex Subunit MED16 Controls Endoreduplication and Cell Growth in Arabidopsis. Plant Cell 31:1899-1912.

Lyu, J., Wang, D., Duan, P., Liu, Y., Huang, K., Zeng, D., Zhang, L., Dong, G., Li, Y., $\mathrm{Xu}$, R., et al. (2020). Control of Grain Size and Weight by the GSK2-LARGE1/OML4 Pathway in Rice. Plant Cell 32:1905-1918.

Ma, H., Duan, J., Ke, J., He, Y., Gu, X., Xu, T.-H., Yu, H., Wang, Y., Brunzelle, J.S., Jiang, Y., et al. (2017). A D53 repression motif induces oligomerization of TOPLESS corepressors and promotes assembly of a corepressor-nucleosome complex. Sci Adv 3:e1601217.

Mao, H., Sun, S., Yao, J., Wang, C., Yu, S., Xu, C., Li, X., and Zhang, Q. (2010). Linking differential domain functions of the GS3 protein to natural variation of grain size in rice. Proceedings of the National Academy of Sciences of the United States of America 107:19579-19584.

McElroy, D., Zhang, W.G., Cao, J., and Wu, R. (1990). ISOLATION OF AN EFFICIENT ACTIN PROMOTER FOR USE IN RICE TRANSFORMATION. Plant Cell 2:163-171.

Murmu, J., Bush, M.J., DeLong, C., Li, S., Xu, M., Khan, M., Malcolmson, C., Fobert, P.R., Zachgo, S., and Hepworth, S.R. (2010). Arabidopsis basic leucine-zipper transcription factors TGA9 and TGA10 interact with floral glutaredoxins ROXY1 and ROXY2 and are redundantly required for anther development. Plant physiology 154:1492-1504.

Ndamukong, I., Abdallat, A.A., Thurow, C., Fode, B., Zander, M., Weigel, R., and 
Gatz, C. (2007). SA-inducible Arabidopsis glutaredoxin interacts with TGA factors and suppresses JA-responsive PDF1.2 transcription. Plant J 50:128-139.

Nijhawan, A., Jain, M., Tyagi, A.K., and Khurana, J.P. (2008). Genomic survey and gene expression analysis of the basic leucine zipper transcription factor family in rice. Plant Physiol 146:333-350.

Pauwels, L., Barbero, G.F., Geerinck, J., Tilleman, S., Grunewald, W., Perez, A.C., Chico, J.M., Bossche, R.V., Sewell, J., Gil, E., et al. (2010). NINJA connects the co-repressor TOPLESS to jasmonate signalling. Nature 464:788-791.

Pham, V.N., Kathare, P.K., and Huq, E. (2018). Dynamic regulation of PIF5 by COP1-SPA complex to optimize photomorphogenesis in Arabidopsis. Plant $\mathrm{J}$ 96:260-273.

Qi, P., Lin, Y.S., Song, X.J., Shen, J.B., Huang, W., Shan, J.X., Zhu, M.Z., Jiang, L., Gao, J.P., and Lin, H.X. (2012). The novel quantitative trait locus GL3.1 controls rice grain size and yield by regulating Cyclin-T1;3. Cell Res 22:1666-1680.

Running, M.P., and Meyerowitz, E.M. (1996). Mutations in the PERIANTHIA gene of Arabidopsis specifically alter floral organ number and initiation pattern. Development 122:1261-1269.

Sharma, R., Priya, P., and Jain, M. (2013). Modified expression of an auxin-responsive rice CC-type glutaredoxin gene affects multiple abiotic stress responses. Planta 238:871-884.

Shi, C., Ren, Y., Liu, L., Wang, F., Zhang, H., Tian, P., Pan, T., Wang, Y., Jing, R., Liu, T., et al. (2019). Ubiquitin Specific Protease 15 Has an Important Role in Regulating Grain Width and Size in Rice. Plant physiology 180:381-391.

Shim, K.C., Kim, S.H., Jeon, Y.A., Lee, H.S., Adeva, C., Kang, J.W., Kim, H.J., Tai, T.H., and Ahn, S.N. (2020). A RING-Type E3 Ubiquitin Ligase, OsGW2, Controls Chlorophyll Content and Dark-Induced Senescence in Rice. Int J Mol Sci 21:1704.

Si, L., Chen, J., Huang, X., Gong, H., Luo, J., Hou, Q., Zhou, T., Lu, T., Zhu, J., Shangguan, Y., et al. (2016). OsSPL13 controls grain size in cultivated rice. Nat Genet 48:447-456.

Song, X.J., Huang, W., Shi, M., Zhu, M.Z., and Lin, H.X. (2007). A QTL for rice grain width and weight encodes a previously unknown RING-type E3 ubiquitin ligase. Nat Genet 39:623-630.

Stroher, E., and Millar, A.H. (2012). The biological roles of glutaredoxins. Biochem J 446:333-348.

Sun, S., Wang, L., Mao, H., Shao, L., Li, X., Xiao, J., Ouyang, Y., and Zhang, Q. (2018). A G-protein pathway determines grain size in rice. Nat Commun 9:851.

Tian, T., Liu, Y., Yan, H., You, Q., Yi, X., Du, Z., Xu, W., and Su, Z. (2017). agriGO v2.0: a GO analysis toolkit for the agricultural community, 2017 update. Nucleic Acids Res 45:W122-W129.

Uhrig, J.F., Huang, L.J., Barghahn, S., Willmer, M., Thurow, C., and Gatz, C. (2017). CC-type glutaredoxins recruit the transcriptional co-repressor TOPLESS to TGA-dependent target promoters in Arabidopsis thaliana. Biochim Biophys Acta 1860:218-226.

Wang, S., Wu, K., Qian, Q., Liu, Q., Li, Q., Pan, Y., Ye, Y., Liu, X., Wang, J., Zhang, 
J., et al. (2017). Non-canonical regulation of SPL transcription factors by a human OTUB1-like deubiquitinase defines a new plant type rice associated with higher grain yield. Cell Res 27:1142-1156.

Wang, S., Wu, K., Yuan, Q., Liu, X., Liu, Z., Lin, X., Zeng, R., Zhu, H., Dong, G., Qian, Q., et al. (2012). Control of grain size, shape and quality by OsSPL16 in rice. Nat Genet 44:950-954.

Wang, Y., Xiong, G., Hu, J., Jiang, L., Yu, H., Xu, J., Fang, Y., Zeng, L., Xu, E., Xu, J., et al. (2015). Copy number variation at the GL7 locus contributes to grain size diversity in rice. Nat Genet 47:944-948.

Wang, Z., Li, N., Jiang, S., Gonzalez, N., Huang, X., Wang, Y., Inze, D., and Li, Y. (2016). SCF(SAP) controls organ size by targeting PPD proteins for degradation in Arabidopsis thaliana. Nat Commun 7:1-11.

Wang, Z., Xing, S., Birkenbihl, R.P., and Zachgo, S. (2009). Conserved functions of Arabidopsis and rice CC-type glutaredoxins in flower development and pathogen response. Mol Plant 2:323-335.

Wu, Q., Yang, J., Cheng, N., Hirschi, K.D., White, F.F., and Park, S. (2017). Glutaredoxins in plant development, abiotic stress response, and iron homeostasis: From model organisms to crops. Environmental and Experimental Botany 139:91-98.

Xia, D., Zhou, H., Liu, R., Dan, W., Li, P., Wu, B., Chen, J., Wang, L., Gao, G., Zhang, Q., et al. (2018). GL3.3, a Novel QTL Encoding a GSK3/SHAGGY-like Kinase, Epistatically Interacts with GS3 to Produce Extra-long Grains in Rice. Mol Plant 11:754-756.

Xia, T., Chen, H., Dong, S., Ma, Z., Ren, H., Zhu, X., Fang, X., and Chen, F. (2020). OsWUS promotes tiller bud growth by establishing weak apical dominance in rice. Plant J 104:1635-1647.

Xia, T., Li, N., Dumenil, J., Li, J., Kamenski, A., Bevan, M.W., Gao, F., and Li, Y. (2013). The ubiquitin receptor DA1 interacts with the E3 ubiquitin ligase DA2 to regulate seed and organ size in Arabidopsis. Plant Cell 25:3347-3359.

Xing, S., and Zachgo, S. (2008). ROXY1 and ROXY2, two Arabidopsis glutaredoxin genes, are required for anther development. Plant J 53:790-801.

Xu, F., Tang, J., Gao, S., Cheng, X., Du, L., and Chu, C. (2019). Control of rice pre-harvest sprouting by glutaredoxin-mediated abscisic acid signaling. Plant $\mathrm{J}$ 100:1036-1051.

Xu, R., Duan, P., Yu, H., Zhou, Z., Zhang, B., Wang, R., Li, J., Zhang, G., Zhuang, S., Lyu, J., et al. (2018a). Control of Grain Size and Weight by the OsMKKK10-OsMKK4-OsMAPK6 Signaling Pathway in Rice. Molecular plant 11:860-873.

Xu, R., Yu, H., Wang, J., Duan, P., Zhang, B., Li, J., Li, Y., Xu, J., Lyu, J., Li, N., et al. (2018b). A mitogen-activated protein kinase phosphatase influences grain size and weight in rice. Plant $\mathrm{J}$.

Ying, J.Z., Ma, M., Bai, C., Huang, X.H., Liu, J.L., Fan, Y.Y., and Song, X.J. (2018). TGW3, a Major QTL that Negatively Modulates Grain Length and Weight in Rice. Mol Plant 11:750-753. 
Yoshida, A., Ohmori, Y., Kitano, H., Taguchi-Shiobara, F., and Hirano, H.Y. (2012). ABERRANT SPIKELET AND PANICLE1, encoding a TOPLESS-related transcriptional co-repressor, is involved in the regulation of meristem fate in rice. Plant J 70:327-339.

Zander, M., Chen, S., Imkampe, J., Thurow, C., and Gatz, C. (2012). Repression of the Arabidopsis thaliana jasmonic acid/ethylene-induced defense pathway by TGA-interacting glutaredoxins depends on their C-terminal ALWL motif. Mol Plant 5:831-840.

Zhai, Q., Zhang, X., Wu, F., Feng, H., Deng, L., Xu, L., Zhang, M., Wang, Q., and Li, C. (2015). Transcriptional Mechanism of Jasmonate Receptor COI1-Mediated Delay of Flowering Time in Arabidopsis. Plant Cell 27:2814-2828.

Zhang, Q. (2007). Strategies for developing green super rice. Proc Natl Acad Sci U S A 104:16402-16409.

Zhang, X., Wang, J., Huang, J., Lan, H., Wang, C., Yin, C., Wu, Y., Tang, H., Qian, Q., and Li, J. (2012). Rare allele of OSPPKL1 associated with grain length causes extra-large grain and a significant yield increase in rice. Proc Natl Acad Sci U S A 109:21534-21539.

Zhang, Y., Li, D., Zhang, D., Zhao, X., Cao, X., Dong, L., Liu, J., Chen, K., Zhang, H., Gao, C., et al. (2018). Analysis of the functions of TaGW2 homoeologs in wheat grain weight and protein content traits. Plant J 94:857-866.

Zhang, Y., Su, J., Duan, S., Ao, Y., Dai, J., Liu, J., Wang, P., Li, Y., Liu, B., and Feng, D. (2011). A highly efficient rice green tissue protoplast system for transient gene expression and studying light/chloroplast-related processes. Plant methods 7:1-14.

Zhao, D.S., Li, Q.F., Zhang, C.Q., Zhang, C., Yang, Q.Q., Pan, L.X., Ren, X.Y., Lu, J., Gu, M.H., and Liu, Q.Q. (2018). GS9 acts as a transcriptional activator to regulate rice grain shape and appearance quality. Nat Commun 9:1-14.

Zhou, Z., Bi, G., and Zhou, J.-M. (2018). Luciferase Complementation Assay for Protein-Protein Interactions in Plants. Current protocols in plant biology 3:42-50.

\section{Figure Legends}

Fig. 1. WG1 controls grain width and weight by influencing cell proliferation.

(A) Grains of Zhonghuajing (ZHJ), WG1-OE and proActin:WG1 lines.

(B) Grains of ZHJ, wg1-1, wg1-2 and wg1-3. The wg1-1, wg1-2 and wg1-3 were generated by CRISPR/Cas9-based genome editing.

(C) The gene structure of WG1. The start codon (ATG) and the stop codon (TAG) are indicated. Black box indicates the CDS and white boxes show the 5'- and 3'untranslated regions. The mutation sites of $w g 1-1, w g 1-2$ and $w g 1-3$ are shown.

(D) The WG1 protein. WG1 protein contains catalytic active site motif CCMC, glutathione binding site Glycine (G), L**LL motif and ALWL motif. 
(E-G) Grain width (E), grain length (F) and 1,000-grain weight (G) of ZHJ, WG1-OE and proActin:WG1 lines.

(H-J) Grain width (H), grain length (I) and 1,000-grain weight (J) of ZHJ, wg1-1, wgl-2 and wgl-3.

(K) The outer epidermal cell width of ZHJ, proActin:WG1\#1 and wg1-1 of spikelet hulls in the center part of the mature grains.

(L) The outer epidermal cell number of ZHJ, proActin:WG1\#1 and wg1-1 in the grain-width direction.

Values in $\mathbf{E}$ to $\mathbf{L}$ are means $\pm \mathrm{SD}(\mathrm{n}=55 / 45 / 53 / 55$ for $\mathbf{E}$ and $\mathbf{F}, \mathrm{n}=3$ for $\mathbf{G}$, $\mathrm{n}=54 / 59 / 49 / 51$ for $\mathbf{H}$ and $\mathbf{I}, \mathrm{n}=4$ for $\mathbf{J}, \mathrm{n}=12 / 11 / 14$ for $\mathbf{K}, \mathrm{n}=13 / 12 / 14$ for $\mathbf{L}$ ). $\mathrm{P}$ value is determined using t-test compared with ZHJ in E-L.. ** represents $\mathrm{P} \leq 0.01$ and * represents $\mathrm{P} \leq 0.05$.

Scale bars in $\mathbf{A}$ and $\mathbf{B}$ represent $5 \mathrm{~mm}$.

\section{Fig. 2. Expression and subcellular localization of WG1.}

(A) WG1 expression was investigated using transgenic lines of proWG1:WG1-GUS. GUS activity in proWG1:WG1-GUS was detected in coleoptile, roots, shoot tips and developing panicles. Scale bars represent $1 \mathrm{~cm}$.

(B) Relative quantitative real time -PCR analysis of WG1 expression in young panicles of $1 \mathrm{~cm}$ (YP1), $5 \mathrm{~cm}$ (YP5), $10 \mathrm{~cm}$ (YP10), $15 \mathrm{~cm}$ (YP15) and 20cm (YP20) of ZHJ plants. Values are means $\pm \mathrm{SD}(\mathrm{n}=3)$.

(C) Subcellular localization of WG1-GFP in root cells of transgenic lines of proWG1:WG1-GFP. The proteins are mainly localized to the nuclei. WG1-GFP, DAPI staining, merge and bright field are shown. Scale bars represent $10 \mu \mathrm{m}$.

(D) Grain width of ZHJ, wg1-1 and proWG1:WG1-GFP;wg1-1. proWG1:WG1-GFP;wg1-1 is wg1-1 crossed with proWG1:WG1-GFP. Values are means $\pm \mathrm{SD}(\mathrm{n}=58 / 62 / 49)$. P value is determined using t-test compared with $\mathrm{wg} 1-1$. ** represents $\mathrm{P} \leq 0.01$. 
(A) WG1 interacts with GW2 in yeast cells. The N-terminus of GW2 (aa:1-114) including a RING domain and the C-terminus (aa:94-425) were shown. The indicated construct pairs were co-transformed into yeast strain AH109. Interactions between bait and prey were examined on the control media -2 (SD/-Leu/-Trp) and selective media -4 (SD/-Leu/-Trp/-His/-Ade).

(B) GW2 associates with WG1 in split luciferase complementation assay. N.benthamiana leaves were transformed by injection of Agrobacterium GV3101 cells harboring $G W 2-n L U C$ and $c L U C-W G 1$ plasmids. Strong luciferase complementation was observed for GW2-nLUC and cLUC-WG1 combination, while no obvious signal was observed for the negative controls.

(C) GW2 associates with WG1 in rice. proWG1:WG1-GFP;pro35S:Myc-GW2 and pro35S:GFP;pro35S:Myc-GW2 transgenic rice plants were used to perform co-immunoprecipitation. Total proteins from pro35S:GFP;pro35S:Myc-GW2 (1) and proWG1:WG1-GFP;pro35S:Myc-GW2 (2) plants were isolated and incubated with GFP-Trap-A agarose beads, and precipitates were detected with anti-GFP and anti-Myc antibodies, respectively. Myc-GW2 was detected in the immunoprecipitated WG1-GFP complex. IP, immunoprecipitation; IB, immunoblot; IN, input.

(D) Subcellular fractionation and immunoblot assays. Young seedlings of pro35S:Myc-GW2 plants were used to isolate the nuclear protein fraction $(\mathrm{N})$ and the cytoplasmic protein fraction (C). Immunoblotting was carried out with an antibody against Myc. Bip, a luminal-binding protein, was used as cytoplasmic marker. Histone $\mathrm{H} 4$ was used as nuclear marker.

\section{Fig. 4. GW2 ubiquitinates WG1 and modulates its stability.}

(A) WG1 can be ubiquitinated by GW2 in vitro. MBP-GW2 and FLAG-WG1 fusion proteins were assayed for ubiquitination activity in the presence of GST-E1, E2, and HIS-ubiquitin (HIS-Ub). Ubiquitinated proteins were detected by immunoblotting with anti-FLAG antibody, anti-MBP antibody and anti-Ub antibody, respectively. MBP-GW2 can be self-ubiquitinated (anti-MBP). FLAG-WG1 can be ubiquitinated by MBP-GW2 (anti-FLAG). The black arrowheads indicate MBP-GW2 and 
FLAG-WG1 proteins, and the red braces show ubiquitinated MBP-GW2 and FLAG-WG1 proteins, respectively.

(B) Overexpression of GW2 results in the reduced level of WG1-GFP protein in rice. Total proteins from 10-day old young seedlings of proWG1:WG1-GFP;ZHJ and proWG1:WG1-GFP;pro35S:Myc-GW2 transgenic plants were isolated and subjected to immunoblot assays using anti-GFP and anti-ACTIN (as loading control) antibodies, respectively. Quantification of WG1-GFP protein levels was relative to ACTIN.

(C) The WG1-GFP protein accumulates at higher levels in the gw2-cl mutant. Total proteins from 10-day old young seedlings of proWG1:WG1-GFP;gw2-c1 and proWG1:WG1-GFP;ZHJ were subjected to immunoblot assays using anti-GFP and anti-ACTIN (as loading control) antibodies, respectively. Quantification of WG1-GFP protein levels was relative to ACTIN.

(D) The GW2 gene structure. The start codon (ATG) and the stop codon (TAG) are indicated. Closed boxes indicate the CDS, open boxes show the 5'- and 3'-untranslated regions, and the lines between boxes indicate the intron. The mutation site of $g w 2-c 1$ is shown. The $g w 2-c 1$ was generated by CRISPR/Cas9-based genome editing.

(E) Grains of ZHJ, $g w 2-c 1, w g 1-2$ and $w g 1-2 g w 2-c 1$. Scale bar represents $5 \mathrm{~mm}$.

(F) Grain width of ZHJ, gw2-cl, wgl-2 and $w g 1-2$ gw2-cl. Values are means \pm SD $(\mathrm{n}=74 / 69 / 42 / 62) . * *$ represents $\mathrm{P} \leq 0.01$ which is determined using t-test compared with $\mathrm{ZHJ}$, while red $* *$ represents $\mathrm{P} \leq 0.01$ which is determined using t-test between $g w 2-c 1$ and $w g 1-2 g w 2-c 1$. Three replicates in $\mathbf{B}$ and $\mathbf{C}$.

\section{Fig. 5. WG1 interacts with OsbZIP47 and represses its transcriptional activation} activity.

(A) WG1 interacts with OsbZIP47 in yeast cells. The protein structure of OsbZIP47 is shown. The N-terminus of OsbZIP47 (aa:1-161) including bZIP domain and the C-terminus (aa:139-385) are shown. The indicated construct pairs were co-transformed into yeast strain GOLD. Interactions between bait and prey were 
examined on the control media -2 (SD/-Leu/-Trp) and selective media -4 (SD/-Leu/-Trp/-His/-Ade).

(B) OsbZIP47 associates with WG1 in split luciferase complementation assay. N.benthamiana leaves were transformed by injection of Agrobacterium GV3101 cells harboring $c L U C-O s b Z I P 47$ and WG1-nLUC plasmids. Strong luciferase complementation was observed for WG1-nLUC and cLUC-OsbZIP47 combination, while no obvious signal was observed for the negative controls.

(C) OsbZIP47 associates with WG1 in rice. proWG1:WG1-GFP;pro35S:Myc-OsbZIP47 and pro35S:GFP;pro35S:Myc-OsbZIP47 transgenic rice were used to perform co-immunoprecipitation. Total proteins from pro35S:GFP; pro35S:Myc-OsbZIP47 and proWG1:WG1-GFP;pro35S:Myc-OsbZIP47 (2) young panicles were isolated and incubated with GFP-Trap-A agarose beads and precipitates were detected with anti-GFP and anti-Myc antibodies, respectively. Myc-OsbZIP47 were detected in the immunoprecipitated WG1-GFP complex. IP, immunoprecipitation; IB, immunoblot; IN, input.

(D) WG1 can repress the transcriptional activation activity of OsbZIP47 in rice protoplast assay. 5×GAL4-LUC was used as reporter. pPTRL was used as internal control. The GAL4 DNA-binding domain BD was used as negative control. BD-OsbZIP47, 35S-WG1 and 35S-WG1 $\triangle$ ALWL were used as effectors. $\triangle \mathrm{ALWL}$ represents deletion of ALWL motif. Activation fold of BD-OsbZIP47 on reporter gene expression was revealed by relative LUC activity LUC/REN, and the LUC/REN ratios were normalized to the activity of their respective negative control BD. Values are means $\pm \mathrm{SD}(\mathrm{n}=3)$. $\mathrm{P}$ value is determined using t-test between activation fold of BD-OsbZIP47 and their corresponding negative control BD. Data are representative of six experiments performed independently with similar results.

Fig. 6. OsbZIP47 acts in a common pathway with WG1 to regulate grain width and weight. 
1150 (A) The OsbZIP47 gene structure. The start codon (ATG) and the stop codon (TAG) 1151 are indicated. Closed boxes indicate the CDS, open boxes show the 5'- and 1152 3'-untranslated regions, and the lines between boxes indicate the intron. The mutation 1153 site of osbzip47-cl/c2 is shown. The osbzip47-cl/c2 mutants were generated by 1154 CRISPR/Cas9-based genome editing.

1155 (B) Grains of ZHJ and osbzip47-cl.

1156 (C) Grains of ZHJ and proActin:OsbZIP47 lines. OsbZIP47 was overexpressed in 1157 ZHJ background.

1158 (D) Grains of $w g 1-1$ and wg1-1 osbzip47-c2. wg1-1 osbzip47-c2 was generated by 1159 CRISPR/Cas9-based genome editing in wg1-1 background. The osbzip47-c2 has the 1160 same mutation site as that in osbzip47-c1.

1161 (E-G) Grain width (E), grain length $(\mathbf{F})$ and 1,000-grain weight $(\mathbf{G})$ of ZHJ and 1162 mutant osbzip47-c1.

1163 (H-J) Grain width (H), grain length (I) and 1,000-grain weight (J) of ZHJ and 1164 proActin:OsbZIP47 lines.

1165 (K) Expression levels of OsbZIP47 in 10-day-old seedlings of ZHJ and 1166 proActin:OsbZIP47 lines.

1167 (L, M) Grain width (L) and 1,000-grain weight (M) of wg1-1 and wg1-1 osbzip47-c2.

1168 (N) OsbZIP47 expression was investigated using transgenic lines of proOsbZIP47:OsbZIP47-GUS. GUS activity was detected in roots of young seedlings and different length of developing panicles.

1174 (P) Subcellular localization of OsbZIP47 in root cells of transgenic lines of 1175 proOsbZIP47:OsbZIP47-GFP. The proteins are localized to nuclei. OsbZIP47-GFP green fluorescence in the nuclei, the same cells stained with DAPI, their merged 1177 image and the bright field are shown. Scale bars: $5 \mathrm{~mm}$ in $\mathbf{B}-\mathbf{D}, 1 \mathrm{~cm}$ in $\mathbf{N}$ and $10 \mu \mathrm{m}$ in P. Values in E-M and $\mathbf{O}$ are means $\pm \mathrm{SD}$ (n=61/59 for $\mathbf{E}$ and $\mathbf{F}, \mathrm{n}=3$ for $\mathbf{G}, \mathbf{J}, \mathbf{K}$ and $\mathbf{O}, \mathrm{n}=52 / 55 / 66$ for $\mathbf{H}$ and $\mathbf{I}, \mathrm{n}=53 / 51$ for $\mathbf{L}, \mathrm{n}=4$ for $\mathbf{M})$. $\mathrm{P}$ value in $\mathbf{E}-\mathbf{J}$ is determined 
using t-test compared with ZHJ. P value in $\mathbf{L}-\mathbf{M}$ is determined using t-test compared with $w$ g1-1. ** represents $\mathrm{P} \leq 0.01$, * represents $\mathrm{P} \leq 0.05$.

1182

1183

1184

1185

1186

1187

Fig. 7. Model for the GW2-WG1-OsbZIP47 regulatory module-mediated control of grain width and weight.

The transcription factor OsbZIP47 is a negative regulator of grain width and weight. WG1 is positive regulator of grain width and weight. The growth signals may activate WG1 to repress the transcriptional activation activity of OsbZIP47, resulting in the inhibition of downstream gene expression. The repressive effect of WG1 on OsbZIP47 may be realized possibly by recruiting the transcriptional co-repressor ASP1. GW2 encodes an E3 ubiquitin ligase. When the growth signals become weaker or disappear, GW2 can ubiquitinate WG1 and cause the degradation of WG1, thereby releasing the inhibition of OsbZIP47 transcriptional activity and promoting the transcription of downstream genes. 
A

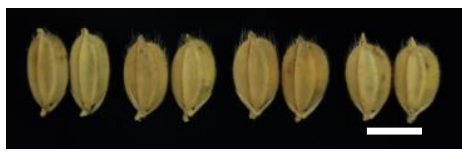

ZHJ WG1-OE $\frac{\# 1 \quad \# 2}{\text { proActin:WG1 }}$

C

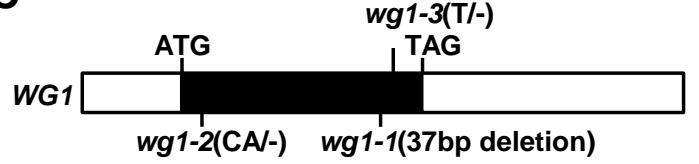

E

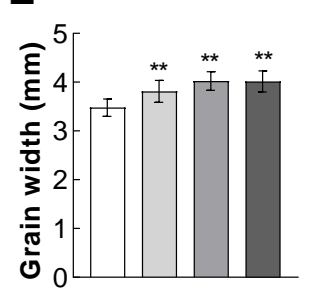

I

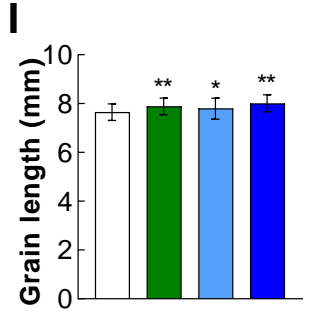

$\mathbf{F}$

- 50bp

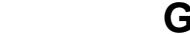

J

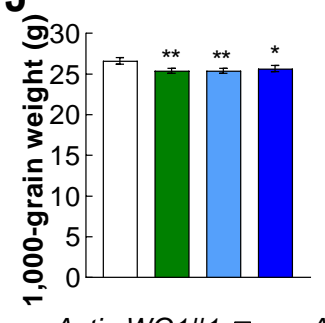

B

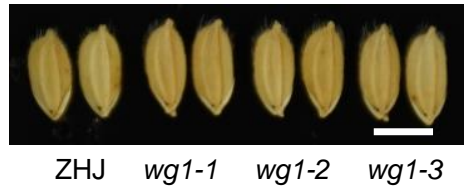

D

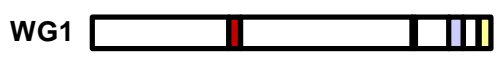

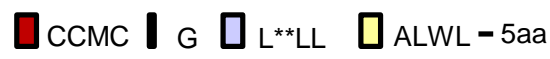

G

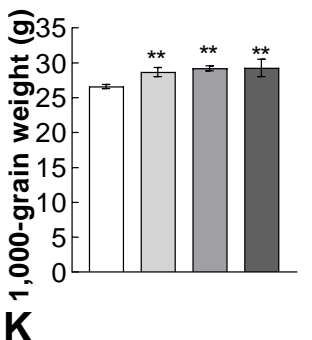

H

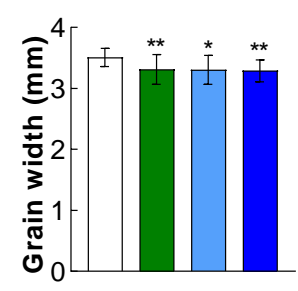

L

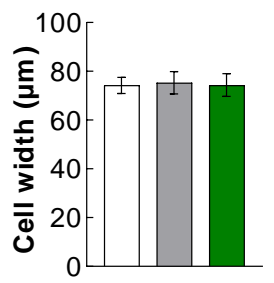

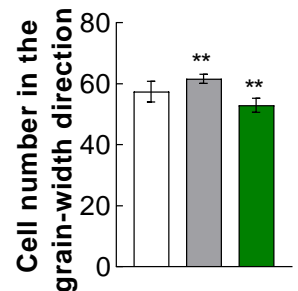

$\square$ ZHJ $\square$ WG1-OE $\square$ proActin:WG1\#1 $\square$ proActin:WG1\#2 $\square$ wg1-1 $\square$ wg1-2 $\square$ wg1-3 
A

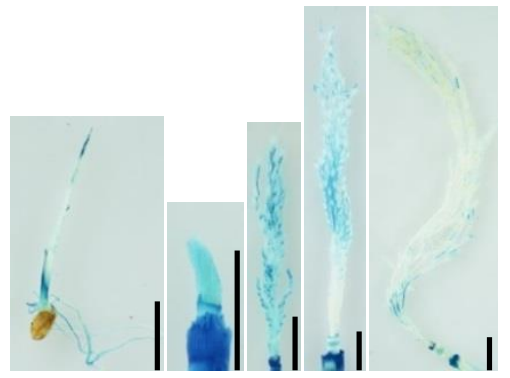

C

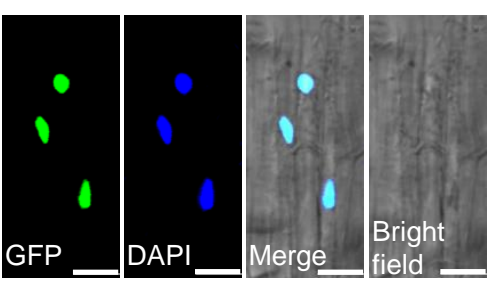

B

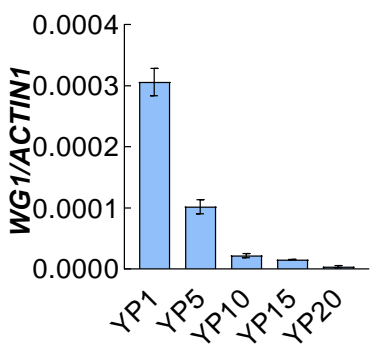

D

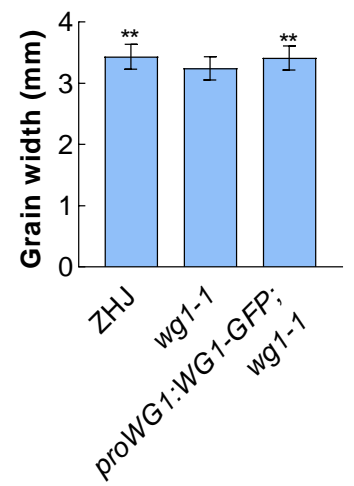


A

\begin{tabular}{|c|c|c|c|}
\hline \multicolumn{2}{|c|}{ GW2(1-425) } & & \\
\hline \multicolumn{2}{|c|}{ GW2(1-114) } & & ING \\
\hline \multicolumn{2}{|c|}{ GW2(94-425) } & & \\
\hline BD & $A D$ & -2 & -4 \\
\hline WG1 & GW2(1-425) & C & \\
\hline WG1 & GW2(1-114) & $\mathrm{C}$ & \\
\hline WG1 & GW2(94-425) & $\mathrm{C}$ & 0 \\
\hline WG1 & - & $\bar{C}$ & \\
\hline- & GW2(1-425) & C & \\
\hline - & GW2(1-114) & $\bar{C}$ & \\
\hline - & GW2(94-425) & & \\
\hline
\end{tabular}

C

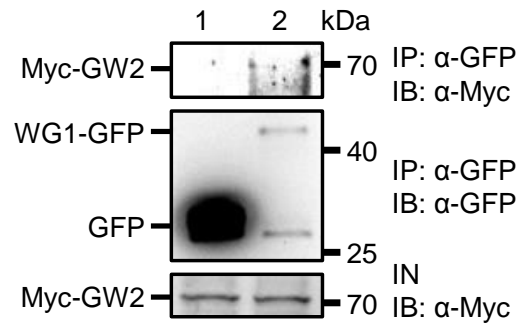

B

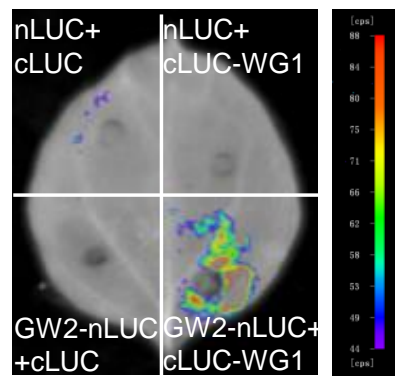

D

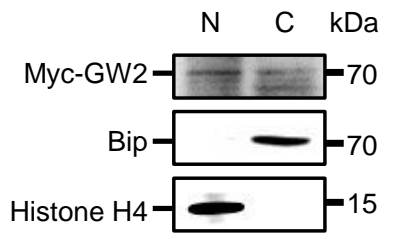


A

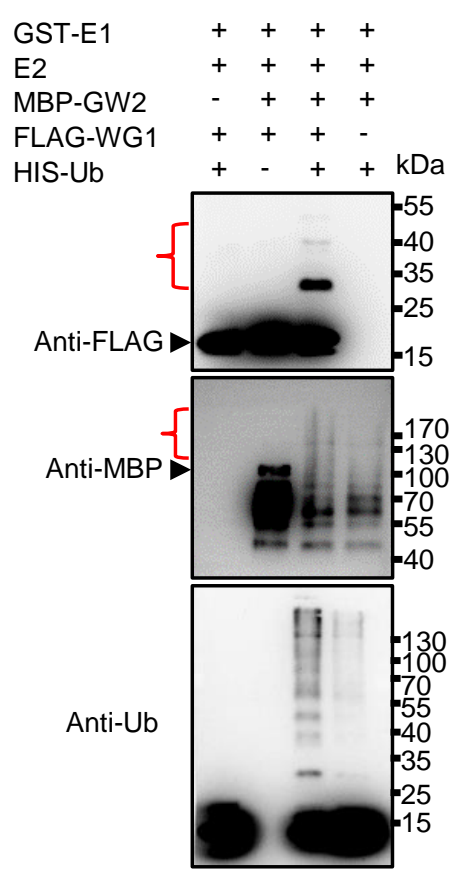

B

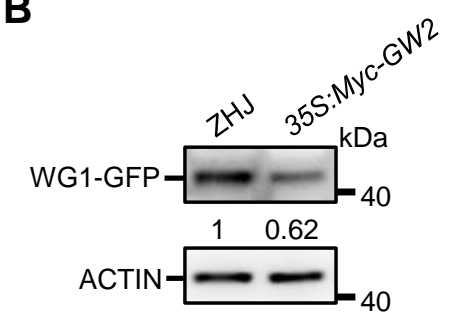

D

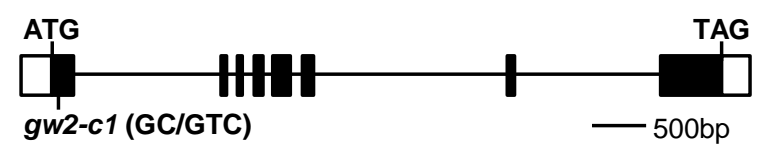

F

E
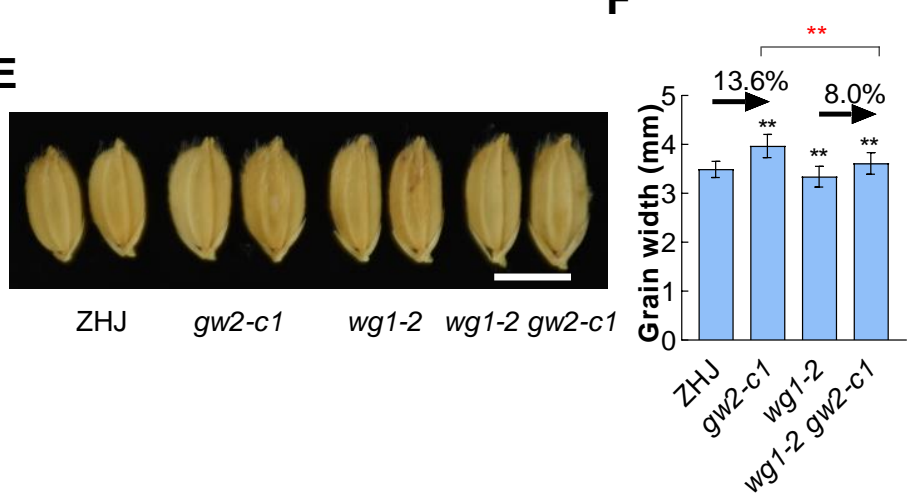
A

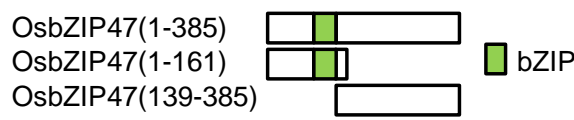

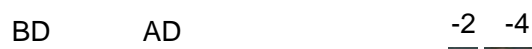

WG1 OsbZIP47(1-385)

WG1 OsbZIP47(1-161)

WG1 OsbZIP47(139-385) D०0

WG1

- $\quad$ OsbZIP47(1-385)

- $\quad$ OsbZIP47(1-161)

- $\quad$ OsbZIP47(139-385)

C

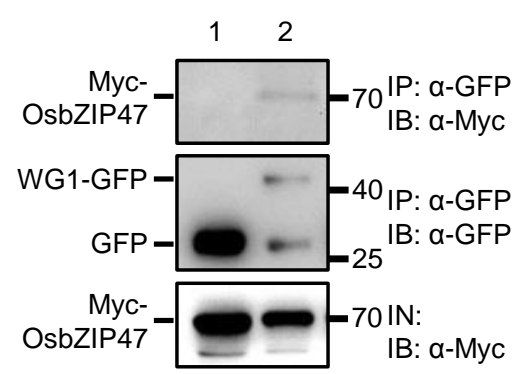

B

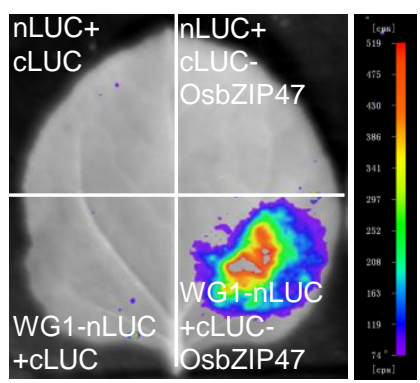

D

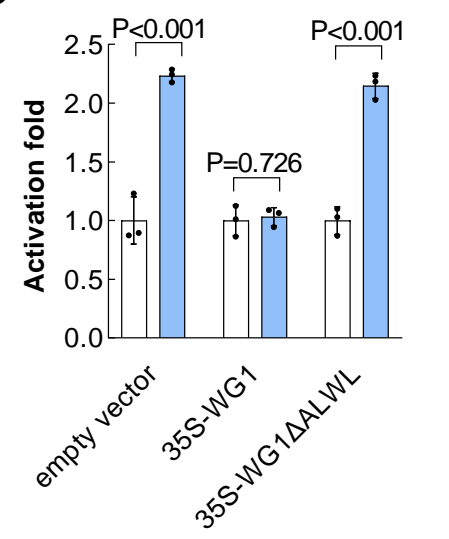

$\square$ BD

BD-OsbZIP47

Effectors:

BD-OsbZIP47;

35S-WG1;

35S-WG1 $\triangle$ ALWL

CK: BD

Reporter:

$5 \times$ GAL4-LUC 
A
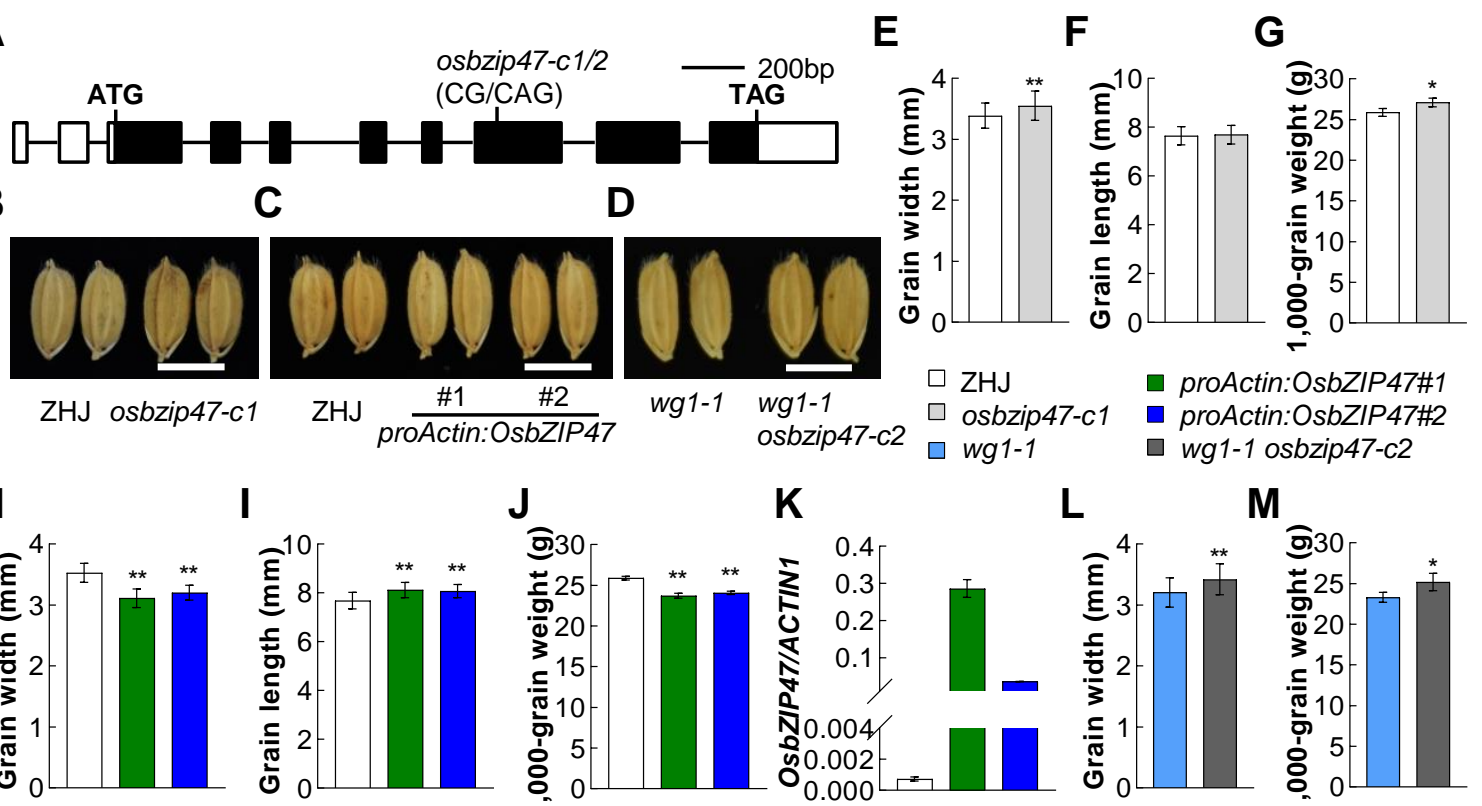

$\mathbf{J}$

K

L

- proActin:OsbZIP47\#2

$\square w g 1-1$

— wg1-1 osbzip47-c2

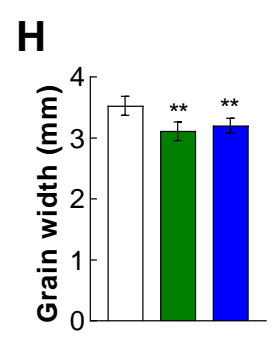

N

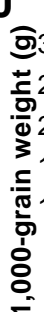

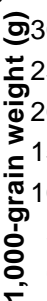

0
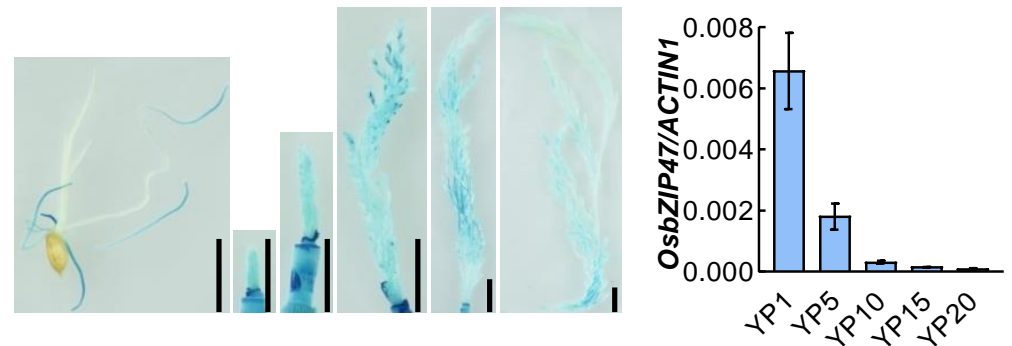

L $\quad \mathbf{M}$

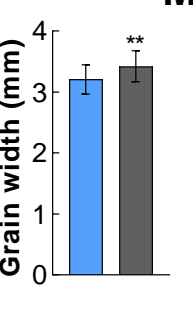

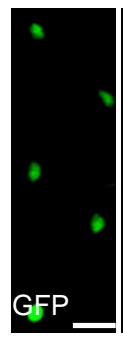
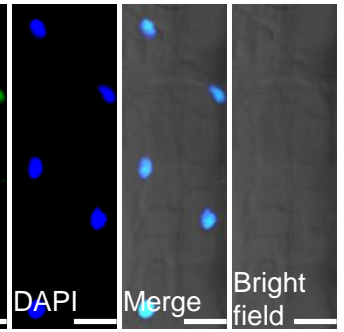


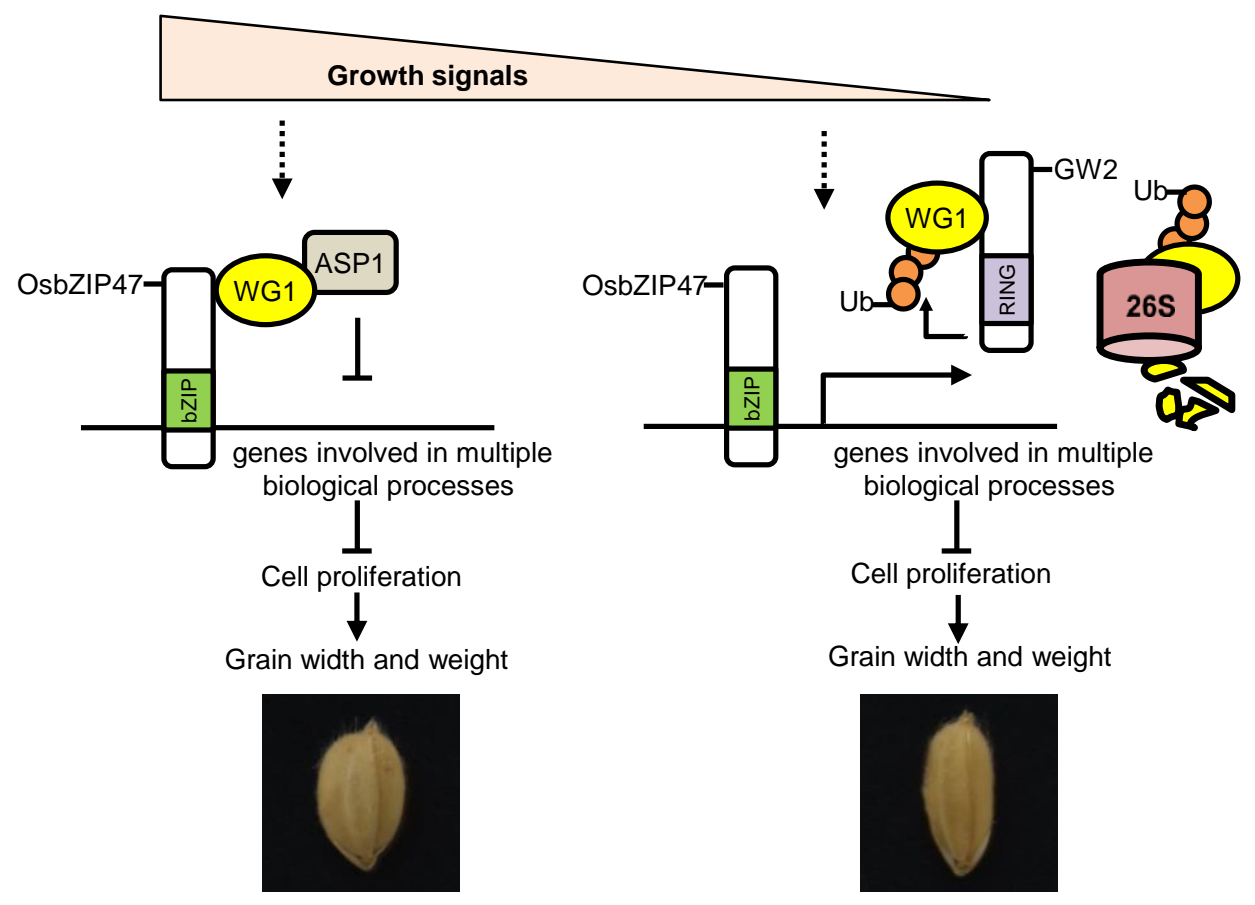


A

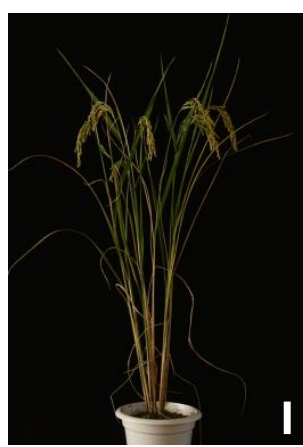

$\mathrm{ZHJ}$

B

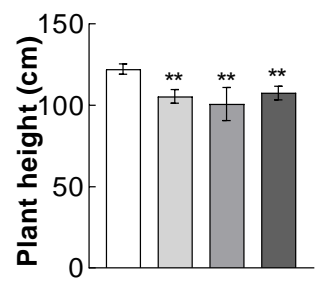

D

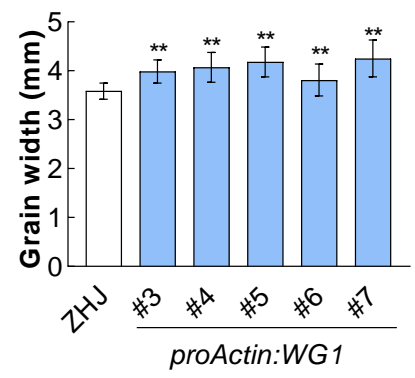

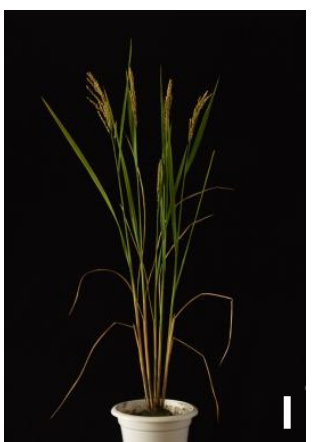

WG1-OE

C

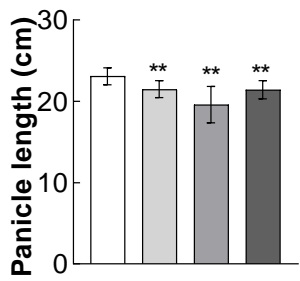

$\square \mathrm{ZHJ}$

$\square$ WG1-OE

$\square$ proActin:WG1\#1

$\square$ proActin:WG1\#2

E

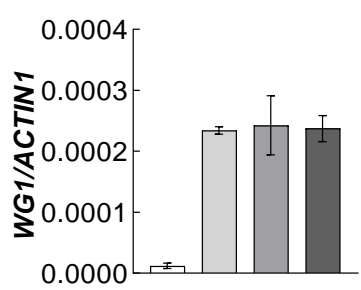

Supplementary Figure 1. Phenotypes of WG1-OE and proActin:WG1 lines.

(A) Mature plant morphology of ZHJ, WG1-OE and proActin:WG1 \#1 plants.

(B, C) Plant height (B) and panicle length (C) of ZHJ, WG1-OE and proActin:WG1 lines.

(D) Grain width of ZHJ and proActin:WG1 lines.

(E) Relative expression levels of WG1 in ZHJ, WG1-OE and proActin:WG1 lines.

Values in $\mathbf{B}(n=10 / 7 / 9 / 7), \mathbf{C}(n=10 / 7 / 8 / 7), \mathbf{D}(n=89 / 71 / 84 / 54 / 97 / 65)$ and $\mathbf{E}(n=3)$ are means $\pm S D$. $P$ value is determined using t-test compared with $\mathrm{ZHJ}$ in $\mathbf{B}-\mathbf{D} .{ }^{* *}$ represents $\mathrm{P} \leq 0.01$. * represents $\mathrm{P} \leq 0.05$. Scale bars in A represent $10 \mathrm{~cm}$. 


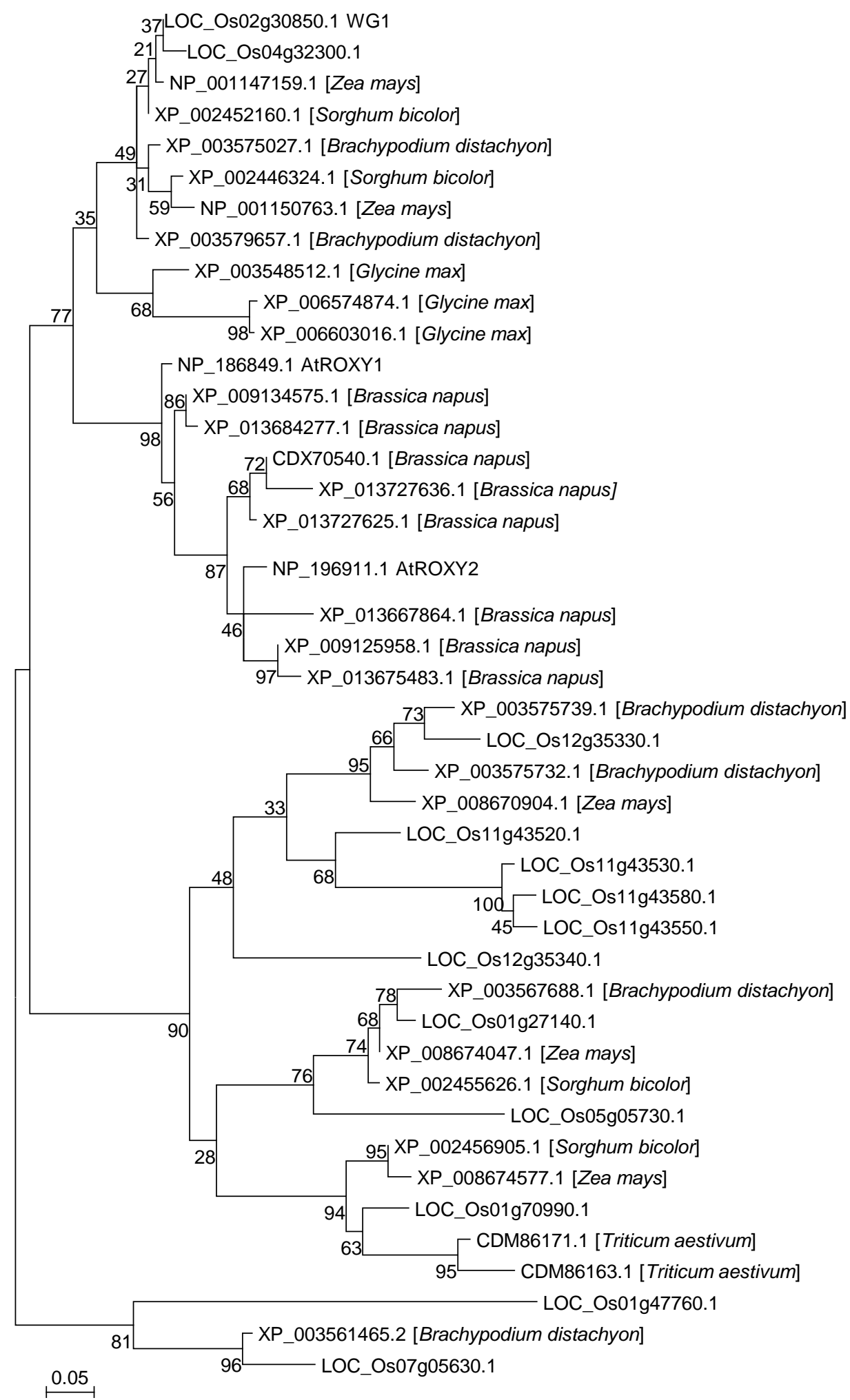

\section{Supplementary Figure 2. Phylogenetic tree of WG1 and its homologs.}

Glutaredoxin(GRX) proteins are ubiquitously present in monocots and dicots. Protein sequences of WG1 homologs ( $>65 \%$ homology, from NBCl) in different species including monocot Oryza sativa, Brachypodium distachyon, Triticum aestivum, Zea mays, Sorghum bicolor and dicot Arabidopsis thaliana, Brassica napus and Glycine max were used to conduct the alignment in ClustalW. A Maximum Likelihood-based tree was generated using MEGA 5.0. Branch lengths are proportional to the phylogenetic distances. Bootstrap testing was conducted with 1000 replicates. Values are indicated at the nodes of branches. 


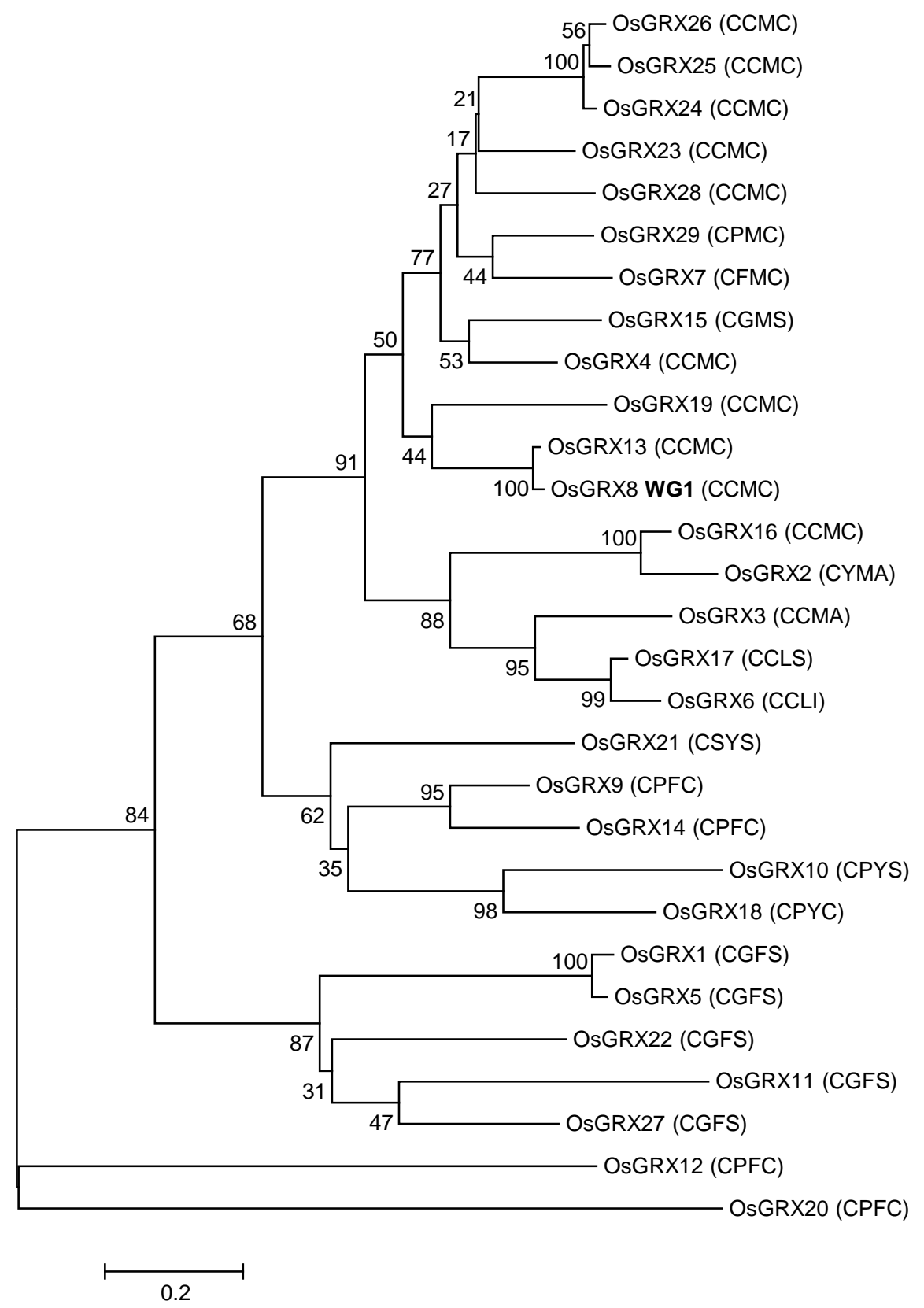

\section{Supplementary Figure 3. Phylogenetic tree of WG1 and its homologs in rice (Oryza sativa L.).}

Glutaredoxin (GRX) proteins could be classified into four classes, namely CC-, CGFS-, CPYC- and GRL-type in rice. We selected 29 CC-type, CGFS-type and CPYC-type GRX sequences for an alignment in ClustalW. A neighbour-joining (NJ)-based tree on Poisson model was generated using MEGA 5.0. Branch lengths are proportional to the phylogenetic distances. Bootstrap testing was conducted with 1000 replicates. Values are indicated at the nodes of branches. Their catalytic active site motifs are given in bracket after gene name. 
A

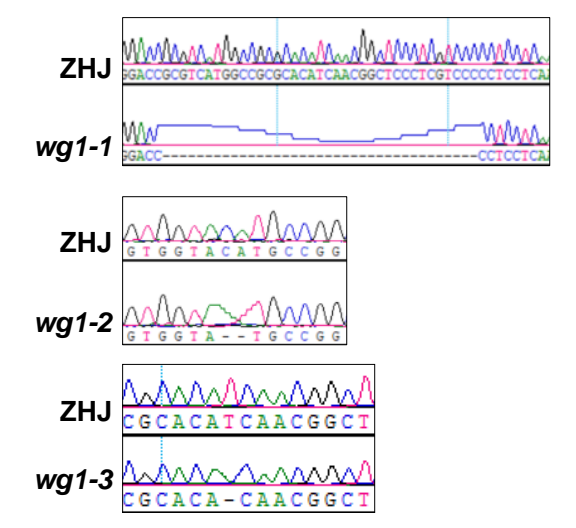

E

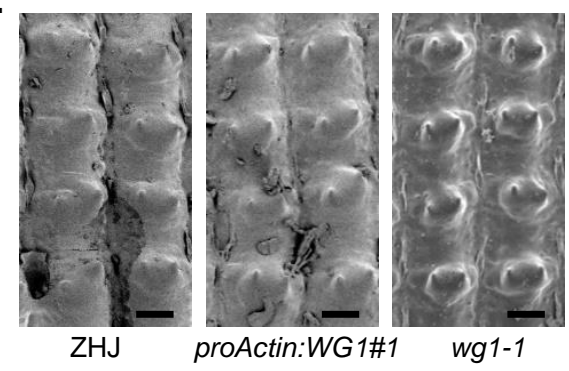

B
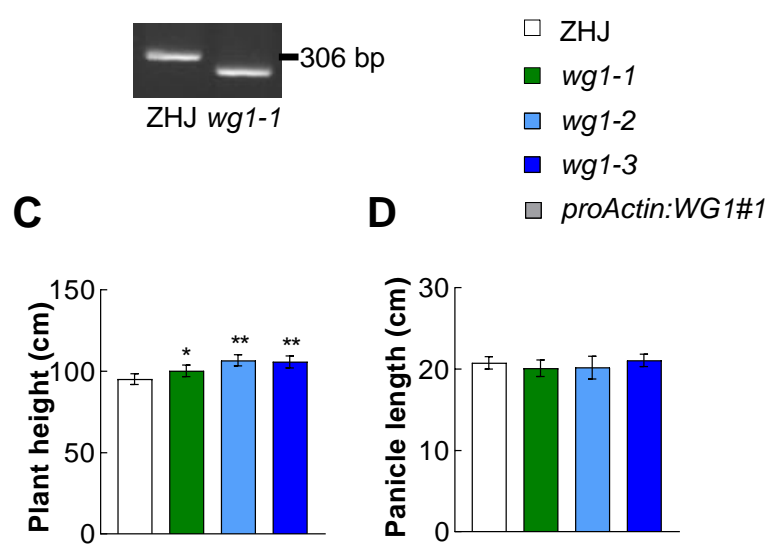

$\mathbf{F}$

G

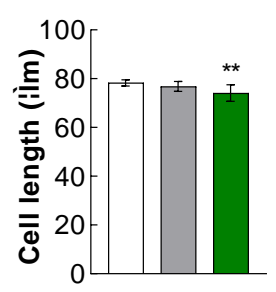

\section{Supplementary Figure 4. Identification of wg1-1, wg1-2 and wg1-3.}

(A) Chromatogram of PCR products inclusive WG1 mutation sites from mutants wg1-1, wg1-2 and wg1-3 are shown. 37-bp deletion in wg1-1, 2-bp deletion in wg1-2 and 1-bp deletion in wg1-3 were detected, respectively.

(B) Primers were developed according to the wg1-1 mutation. PCR products from ZHJ and wg1-1 genomic DNA were separable.

(C, D) Plant height (C) and panicle length (D) of ZHJ, wg1-1, wg1-2 and wg1-3.

(E) Scanning electron microscope images of the outer epidermal cells in spikelet hulls of mature grains. ZHJ (left), proActin:WG1\#1 (middle) and wg1-1 (right). Scale bars represent 50 um.

(F) The outer epidermal cell length of ZHJ, proActin:WG1\#1 and wg1-1 of spikelet hulls in the center part of the mature grains.

(G) The calculated cell number in the grain-length direction of ZHJ, proActin:WG1\#1 and wg1-1 by grain length dividing cell length.

Values in $\mathbf{C}(n=9 / 7 / 11 / 10), \mathbf{D}(n=9 / 11 / 11 / 8), \mathbf{F}(n=12 / 9 / 10)$ and $\mathbf{G}(n=12 / 9 / 10)$ are means \pm SD. $P$ value is determined using t-test compared with $\mathrm{ZHJ}$. in $\mathrm{C}, \mathrm{D}, \mathrm{F}$ and $\mathrm{G}$ * represents $\mathrm{P} \leq 0.05$, ${ }^{* *}$ represents $\mathrm{P} \leq 0.01$. 
A
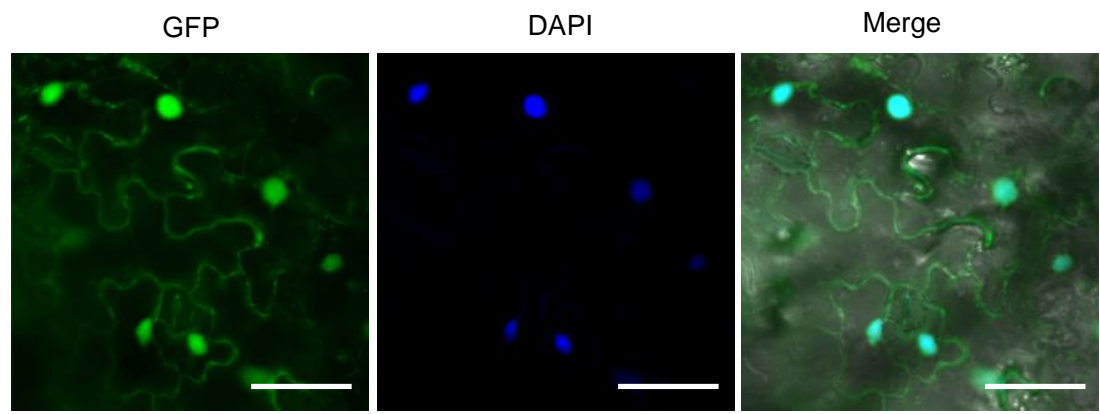

B

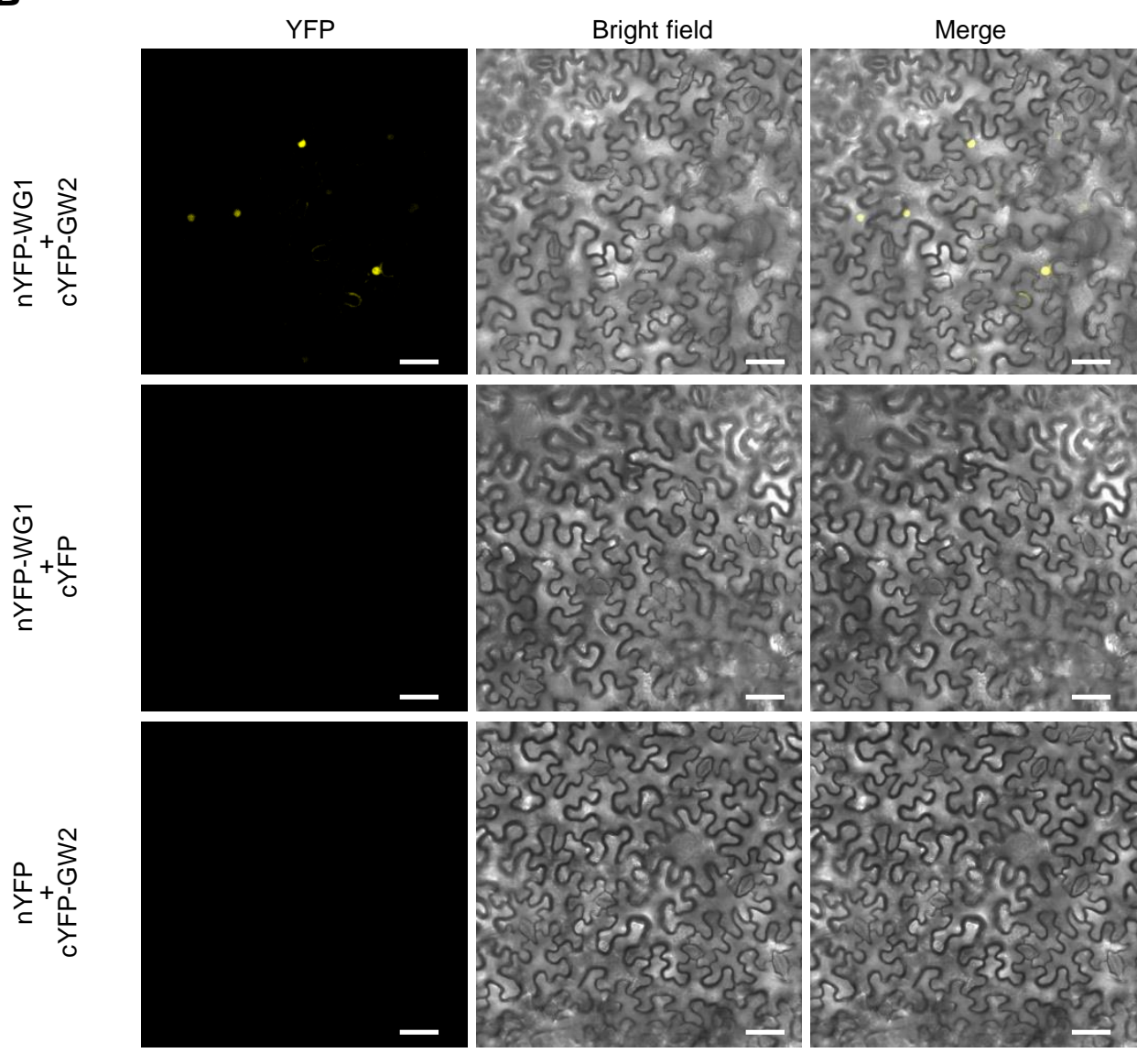

\section{Supplementary Figure 5 . GW2 associates with WG1 in nucleus.}

(A) Subcellular localization of green fluorescent protein (GFP) -GW2 in epidermal cells of N.benthamiana leaves. pro35S:GFP-GW2 were transiently expressed in N.benthamiana leaves. GFPGW2, DAPI staining and merge are shown. Scale bars represent $50 \mu \mathrm{m}$.

(B) Bimolecular fluorescence complementation assays show that WG1 associates with GW2 in nuclei. cYFP-GW2/nYFP-WG1, nYFP-WG1/cYFP and cYFP-GW2/nYFP were co-expressed in leaves of N.benthamiana, respectively. Scale bars represent $50 \mu \mathrm{m}$. 
A

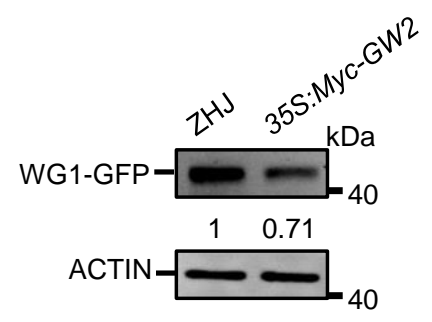

D

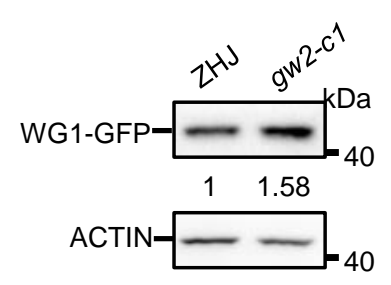

B

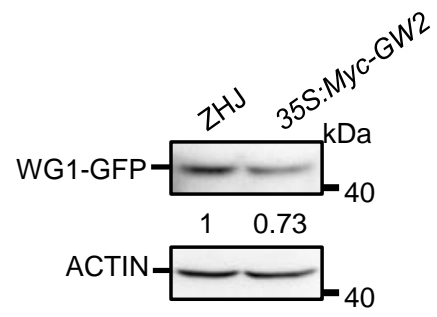

E

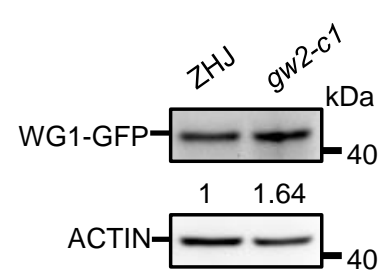

C

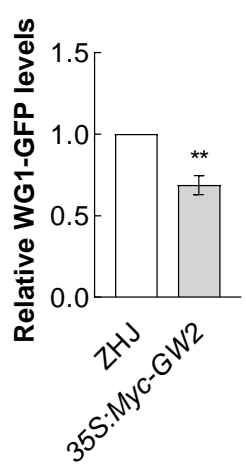

$\mathbf{F}$

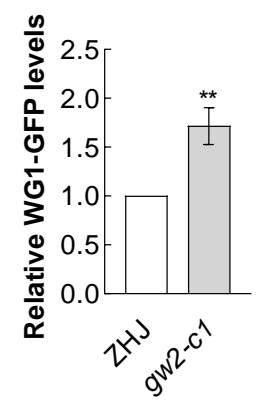

\section{Supplementary Figure 6 . GW2 modulates the stability of WG1 proteins.}

(A-C) Overexpression of GW2 results in the reduced levels of WG1 proteins. Total proteins from 10-day old young seedlings of proWG1:WG1-GFP;ZHJ and proWG1:WG1-GFP;pro35S:Myc-GW2 transgenic plants were isolated and subjected to immunoblot assays using anti-GFP and anti-ACTIN (as loading control) antibodies, respectively. Results in Figure 4b were repeated twice (A, B). Quantification of WG1-GFP protein levels was relative to ACTIN. Band intensities of triplicate repeats (Figure 4B; Supplementary Figure 6A, B) were quantified by the Quantity One program $(n=3)$. Relative levels of WG1-GFP proteins were shown (C). P value is determined using t-test compared with $\mathrm{ZHJ}$. ** represents $\mathrm{P} \leq 0.01$.

(D-F) The WG1-GFP proteins accumulate at higher levels in the gw2-c1 mutant. Total proteins from 10-day old young seedlings of proWG1:WG1-GFP;gW2-c1 and proWG1:WG1-GFP;ZHJ were subjected to immunoblot assays using anti-GFP and anti-ACTIN (as loading control) antibodies, respectively. Results in Figure 4c were repeated twice (D, E). Quantification of WG1-GFP protein levels was relative to ACTIN. Band intensities of triplicate repeats (Figure 4C; Supplementary Figure 6D, E) were quantified by the Quantity One program $(n=3)$. Relative levels of WG1-GFP proteins were shown $(\mathbf{F})$. P value is determined using t-test compared with ZHJ. ** represents $\mathrm{P} \leq 0.01$. 
A
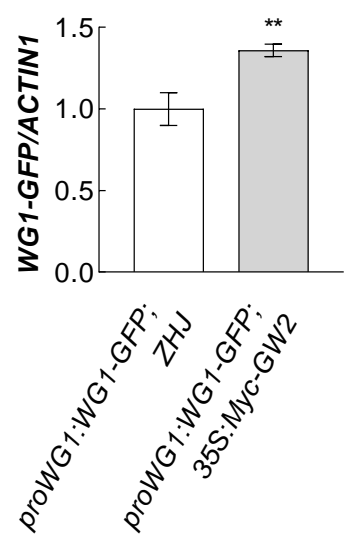

B

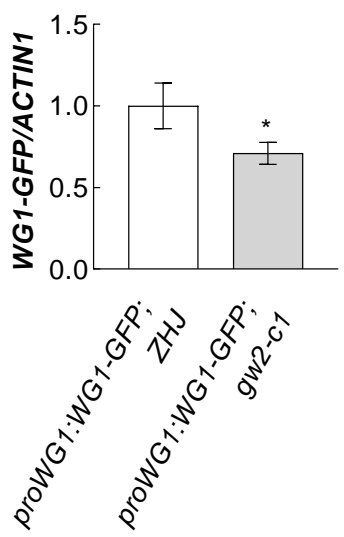

Supplementary Figure 7. mRNA expression levels of WG1-GFP in ZHJ, 35S:Myc-GW2 and gw2-c1 background.

(A) Relative quantitative real time -PCR analysis of WG1-GFP in proWG1:WG1-GFP;ZHJ and proWG1:WG1-GFP;pro35S:Myc-GW2 10-day old seedlings $(\mathrm{n}=3)$.

(B) Relative quantitative real time -PCR analysis of WG1-GFP in proWG1:WG1-GFP;ZHJ and proWG1:WG1-GFP;gw2-c1 10-day old seedlings $(\mathrm{n}=3)$. Values in $\mathbf{A}$ and $\mathbf{B}$ are given as means \pm $S D$ relative to their respective controls, set at 1.

$\mathrm{P}$ value is determined using t-test compared with proWG1:WG1-GFP;ZHJ in $\mathbf{A}$ and $\mathbf{B}$. ${ }^{*}$ represents $\mathrm{P}$ $\leq 0.05,{ }^{* *}$ represents $\mathrm{P} \leq 0.01$. 
A

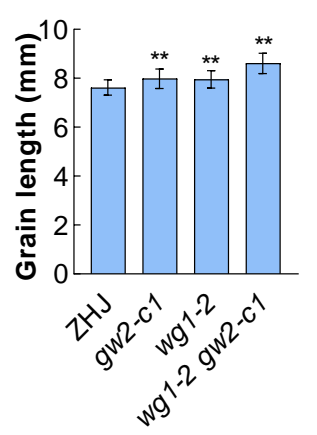

B

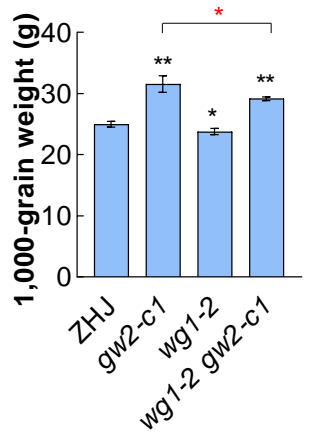

Supplementary Figure 8. Grain phenotypes of ZHJ, gw2-c1, wg1-2 and wg1-2 gw2-c1.

(A, B) Grain length (A) and 1,000-grain weight (B) of ZHJ, gw2-c1, wg1-2 and wg1-2 gw2-c1. Values in $\mathbf{A}(n=74 / 69 / 42 / 62)$ and $\mathbf{B}(n=3)$ are means $\pm S D$. ${ }^{* * *}$ represents $P \leq 0.05 / 0.01$ which is determined using t-test compared with $\mathrm{ZHJ}$, while * represents $\mathrm{P} \leq 0.05$ which is determined using $\mathrm{t}$ test between gw2-c1 and wg1-2 gw2-c1. 


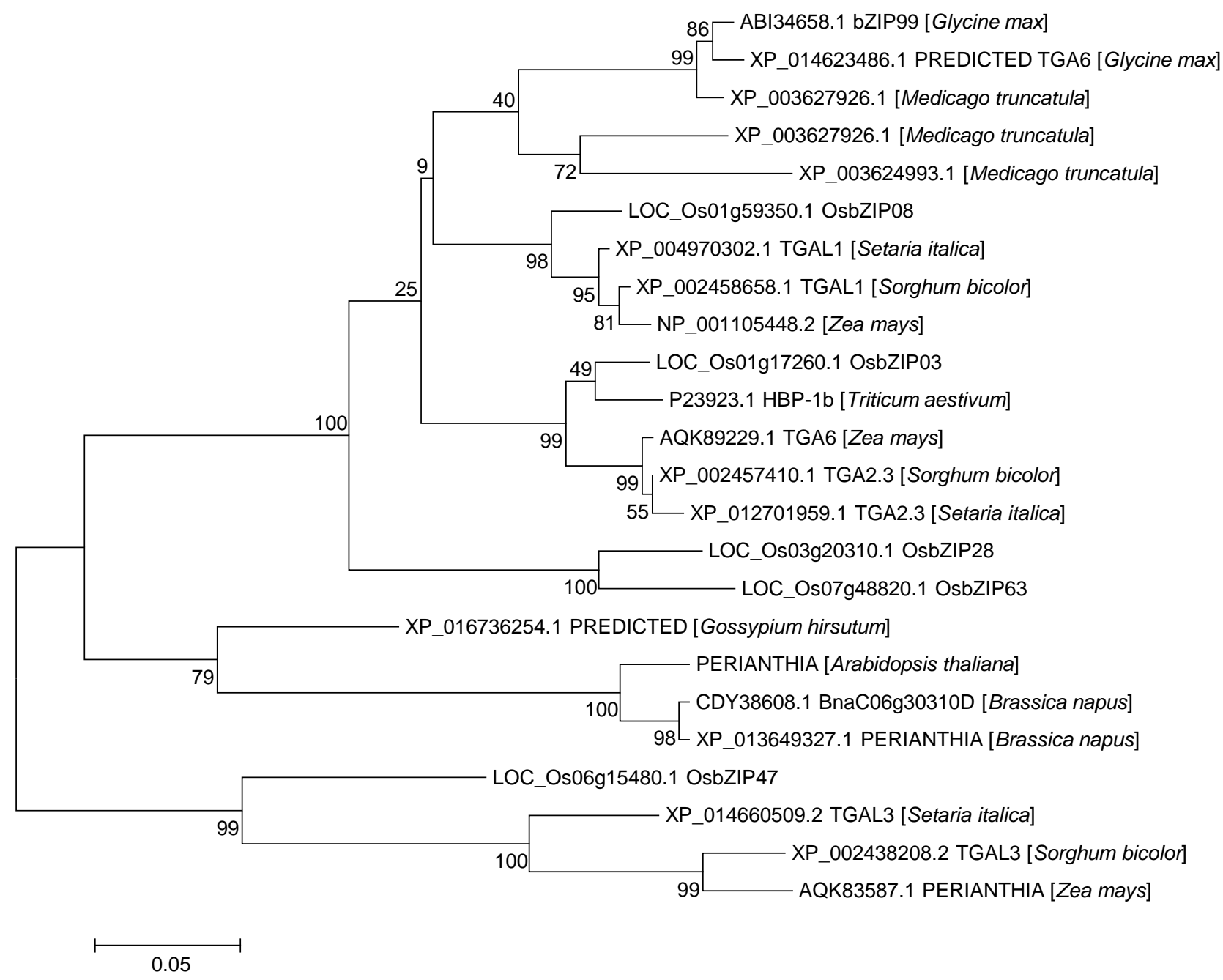

\section{Supplementary Figure 9. Phylogenetic tree of OsbZIP47 and its homologs from different species.}

OsbZIP47 homologs from different species (Oryza sativa, Arabidopsis thaliana, Zea mays, Sorghum bicolor, Setaria italica, Brassica napus, Gossypium hirsutum, Triticum aestivum, Medicago truncatula and Glycine max) were used to conduct alignment in Clustal W. The Maximum Likelihood-based tree was generated using MEGA 5.0. Branch lengths are proportional to the phylogenetic distances. Bootstrap testing was conducted with 1000 replicates. Values are indicated at the nodes of branches. 


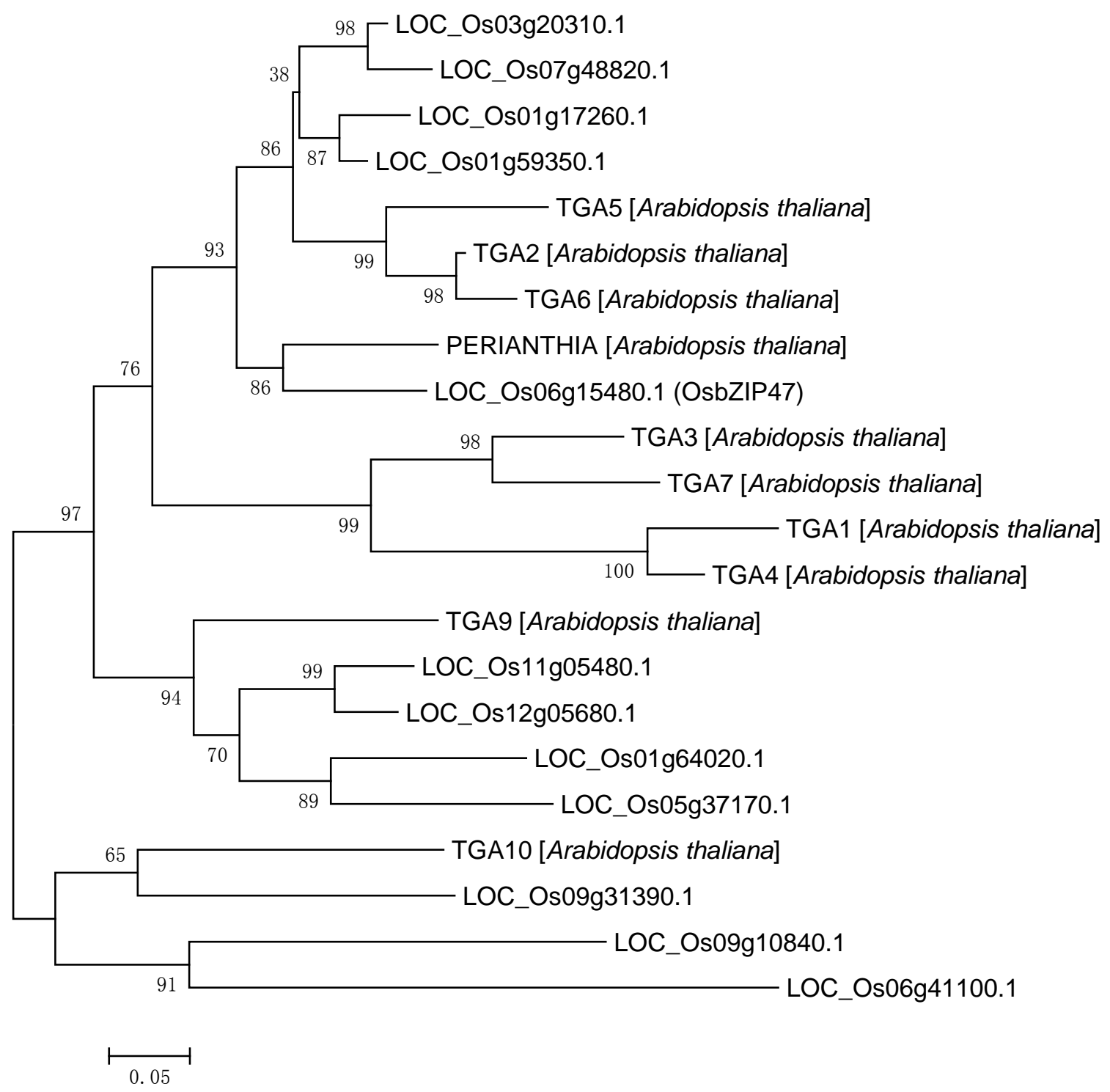

Supplementary Figure 10. Phylogenetic tree of Arabidopsis TGA transcription factors and their homologs in rice.

Protein sequences of Arabidopsis TGA transcription factors (TGA1-7, PERIANTHIA and TGA9-10) and their rice homologs were used to conduct alignment in Clustal W . A neighbour-joining (NJ)-based tree on Poisson model was generated using MEGA 5.2. Branch lengths are proportional to the phylogenetic distances. Bootstrap testing was conducted with 1000 replicates. Values are indicated at the nodes of branches. 


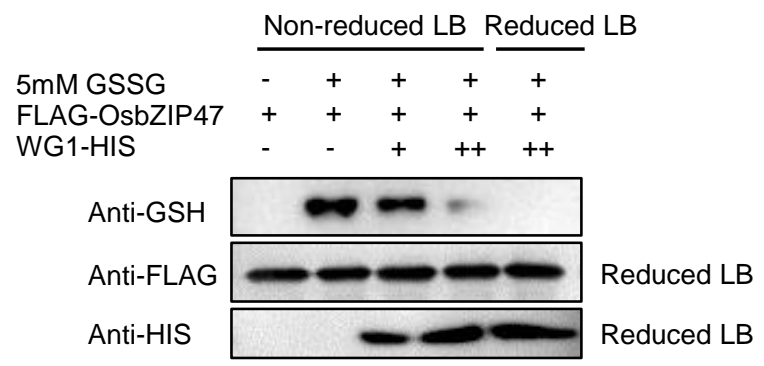

\section{Supplementary Figure 11. The deglutathionylation of OsbZIP47 by WG1 in vitro.}

FLAG-OsbZIP47 was incubated with 5mM GSSG, and different concentrations of HIS-WG1were indicated. FLAG-OsbZIP47 was glutathionylated by 5mM GSSG in vitro. Incubation with increased concentrations of WG1-HIS decreased the glutathionylation of OsbZIP47. The addition of reduced loading buffer removed the glutathionylation of OsbZIP47. The glutathionylation of OsbZIP47 was detected by immunoblotting with anti-Glutathione antibody. Anti-FLAG and Anti-HIS antibodies were used to detect FLAG-OsbZIP47 and WG1-HIS as loading control, respectively. Non-reduced LB: Non-reduced loading buffer. Reduced LB: Reduced loading buffer (with $\beta$-mercaptoethanol and DTT). 
A 1 MAAHQGMAAATAADRFCॄLPRMAAAAAAASQ 30

31 VENWGDSGVIVSSPFTDDTSTDLDDSADKH $\mathbf{6 0}$

61 HLHALVGGGDGGDDAGEQRGADSSAVSKER 90

91 RGDQKMQRRLAQNREAARKSRMRKKAYIQQ 120

121 LESSRSKLMHLEQELQRARQQGIFIATGGS 150

151 GDHGHSIGGNGTLAFDLEYARWLDEHQRHI 180

181 NDLRVALNAQMSDDELCELVDAVMMHYDQV 210

211 FRLKSFATKSDVFHVLSGMWMSPAERFFMW 240

241 LGGFRSSELLKVLASHLEPLTDQQLMGIC్N 270

271 LQQSSQQAEDALSQGMEALQQTLGDTLVSA 300

301 AATVVSGGGGADNVTNYMGQMAIAMAKLTT 330

331 LENFLRQADLLRHQTLQQMHRILTTRQAAR 360

361 ALLVISDYFSRLRALSSLWLARPRD 385

B

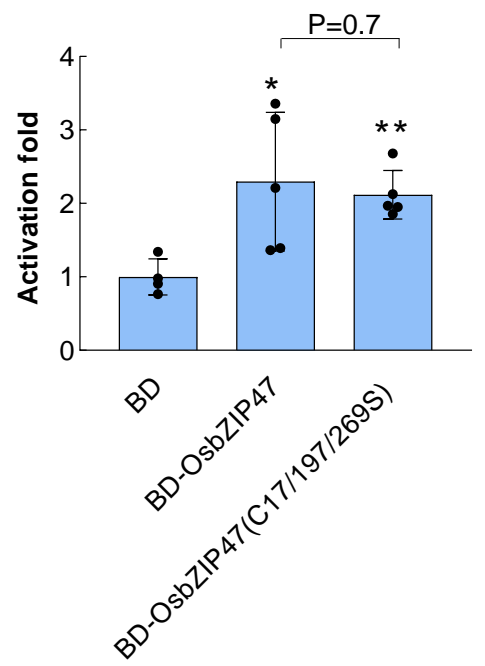

Supplementary Figure 12. The transcriptional activation activity of OsbZIP47 (C17/ C197/269S) in rice protoplast assay.

(A) Protein sequence of OsbZIP47. OsbZIP47 only contains three cysteines that were marked with red Asterisk. Numbers on the right and left side of the sequence represent the corresponding amino position.

(B) DLR assay was conducted to detect transcriptional activation activity of BDOsbZIP47(C17/197/269S) in rice ZHJ protoplasts. 5×GAL4-LUC was used as reporter. pPTRL was used as internal control. The GAL4 DNA-binding domain BD was used as negative control. Activation fold of BD-OsbZIP47/BD-OsbZIP47(C17/197/269S) on reporter gene expression was revealed by relative LUC activity LUC/REN, and the LUC/REN ratios were normalized to the activity of negative control BD. Values are means $\pm S D(n=4 / 5 / 5){ }^{* / * *}$ represents $P \leq 0.05 / 0.01$ which is determined using t-test between activation fold of BD-OsbZIP47/BD-OsbZIP47(C17/197/269S) and BD. Activation fold between BD-OsbZIP47 and BD-OsbZIP47(C17/197/269S) were not different significantly by t-test. Data are representative of three experiments performed independently with similar results. 
A

AD-WG1+BD-ASP1
AD-GW2+BD-ASP1
AD-OsbZIP47+BD-ASP1
AD+BD-ASP1
AD-WG1+BD
AD-GW2+BD
AD-OsbZIP47+BD

B

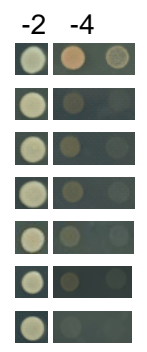

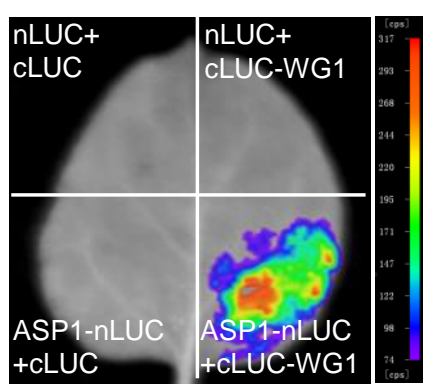

C

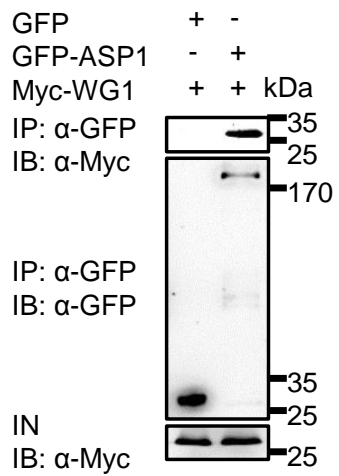

\section{Supplementary Figure 13. WG1 interacts with ASP1.}

(A) WG1 interacts with ASP1 in yeast cells. The indicated construct pairs were co-transformed into yeast strain GOLD. Interactions between bait and prey were examined on the control media -2 (SD/-Leu/-Trp) and selective media -4 (SD/-Leu/-Trp/-His/-Ade, the original concentration and ten percent dilution).

(B) Interaction between ASP1 and WG1 in split luciferase complementation assay. N.benthamiana leaves were transformed by injection of Agrobacterium GV3101 cells harboring ASP1-nLUC and cLUC-WG1 plasmids. Strong luciferase complementation was observed for ASP1-nLUC and CLUC-WG1 combination, while no obvious signal was observed in the negative controls.

(C) WG1 associates with ASP1 in vivo. N.benthamiana leaves were transformed by injection of Agrobacterium GV3101 cells harboring pro35S:Myc-WG1 and pro35S:GFP-ASP1 combination and pro35S:Myc-WG1 and pro35S:GFP combination, respectively. Total proteins were immunoprecipitated with GFP-Trap-A, and the immunoblot was probed with anti-GFP and anti-Myc antibodies, respectively. MycWG1 was detected in the immunoprecipitated GFP-ASP1 complex. IP, immunoprecipitation; IB, immunoblot; IN, input. 
A

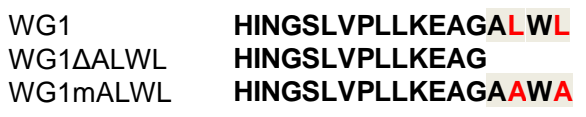

WG1mALWL
B

C
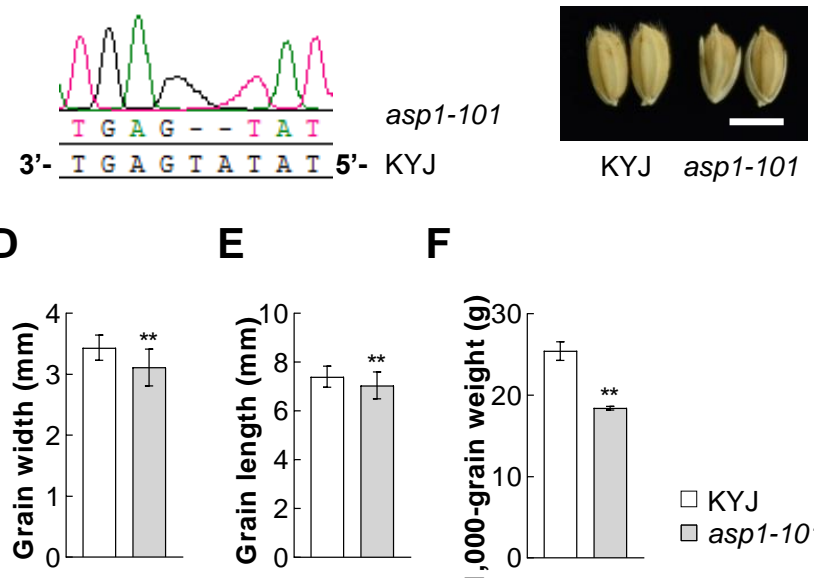

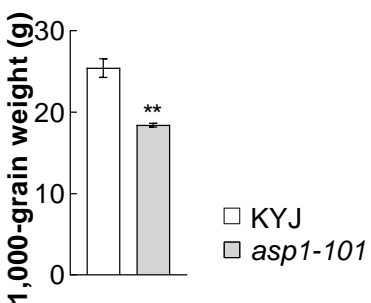

\section{Supplementary Figure 14. WG1 interacts with ASP1 that influences grain size.}

(A) Interaction between WG1 and ASP1 depends on the ALWL motif in yeast cells. C terminal amino acids sequences of WG1, WG1 $\mathrm{WLWL}$ and WG1mALWL are shown. WG1 $\mathrm{WLWL}$ represents deletion of the ALWL motif. WG1mALWL, mutated WG1 in which the Leu residues of the ALWL motif were substituted to Ala residues. The indicated construct pairs were co-transformed into yeast strain AH109. Interactions between bait and prey were examined on the control media -2 (SD/-Leu/-Trp) and selective media -4 (SD/Leu/-Trp/-His/-Ade).

(B) Chromatogram of PCR products inclusive ASP1 mutation sites from mutant asp1-101 is shown. asp1101 was generated from the $\mathrm{y}$-ray irradiation of japonica variety KYJ. 2-bp deletion was detected compared to the wild type KYJ sequence.

(C) Grains of KYJ and asp1-101. Like the previous publications, asp1-101 has elongated glume compared to the wild type.

(D-F) Grain width (D), grain length (E), and 1,000-grain weight (F) of KYJ and asp1-101.

Values in $\mathbf{D}-\mathbf{F}$ are means $\pm S D(n=57 / 48$ for $\mathbf{D}$ and $\mathbf{E}, \mathrm{n}=3$ for $\mathbf{F})$. $P$ value is determined using $t$-test compared with KYJ in $\mathbf{D}-\mathbf{F}$. ${ }^{* *}$ represents $\mathbf{P} \leq 0.01$. Scale bar in $\mathbf{C}$ represents $5 \mathrm{~mm}$. 
A

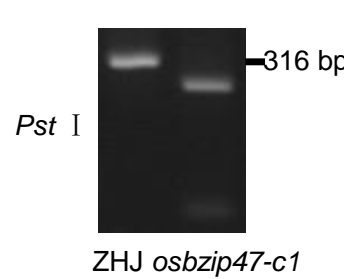

$\mathbf{E}$

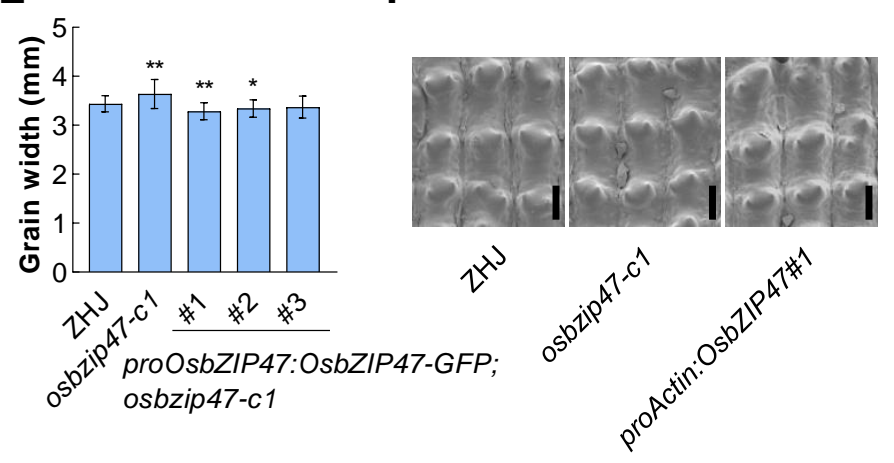

D
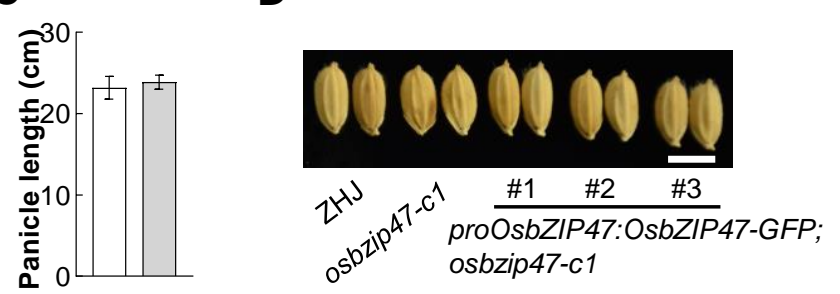

G

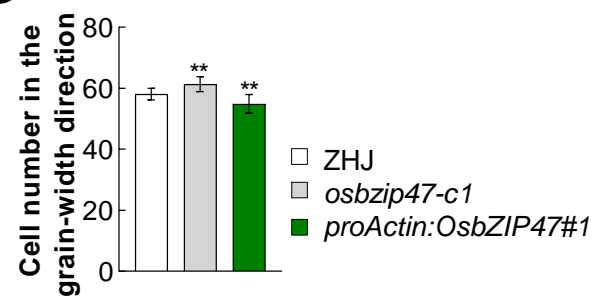

\section{Supplementary Figure 15. Identification of osbzip47-c1 mutant.}

(A) The CAPS marker is developed according to the osbzip47-c1 mutation. The restriction enzyme Pst I was used to digest the PCR product.

(B, C) Plant height (B) and panicle length (C) of ZHJ and osbzip47-c1.

(D) Grains of ZHJ, osbzip47-c1, proOsbZIP47:OsbZIP47-GFP\#1;osbzip47-c1, proOsbZIP47:OsbZIP47GFP\#2;osbzip47-c1 and proOsbZIP47:OsbZIP47-GFP\#3;osbzip47-c1. Scale bar represents $5 \mathrm{~mm}$.

(E) Grain width of ZHJ, osbzip47-c1, proOsbZIP47:OsbZIP47-GFP\#1;osbzip47-c1, proOsbZIP47:OsbZIP47-GFP\#2;osbzip47-c1 and proOsbZIP47:OsbZIP47-GFP\#3;osbzip47-c1. proOsbZIP47:OsbZIP47-GFP;osbzip47-c1 is osbzip47-c1 transformed with proOsbZIP47:OsbZIP47-GFP. (F) Scanning electron microscope images of the outer epidermal cells in spikelet hulls of mature grains. ZHJ (left), osbzip47-c1(middle) and proActin:OsbZIP47\#1 (right). Scale bars represent 40 um.

(G) The outer epidermal cell number of ZHJ, osbzip47-c1 and proActin:OsbZIP47 \#1 in the grain-width direction.

Values in $\mathbf{B}(n=12 / 11), \mathbf{C}(n=10 / 10), \mathbf{E}(n=56 / 60 / 69 / 76 / 79)$ and $\mathbf{G}(n=20 / 19 / 20)$ are means \pm SD. $P$ value is determined using t-test compared with $\mathrm{ZHJ}$ in $\mathrm{B}, \mathrm{C}, \mathrm{E}$ and $\mathrm{G}$. ${ }^{* *}$ represents $\mathrm{P} \leq 0.01$, ${ }^{*}$ represents $\mathrm{P}$ $\leq 0.05$. 
A

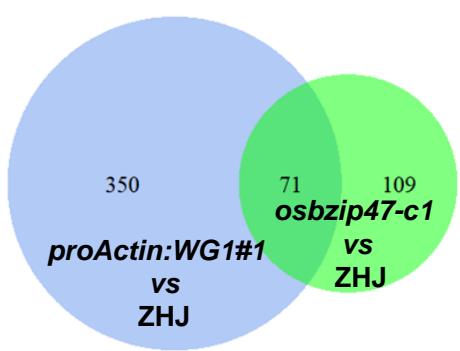

Upregulated genes
B

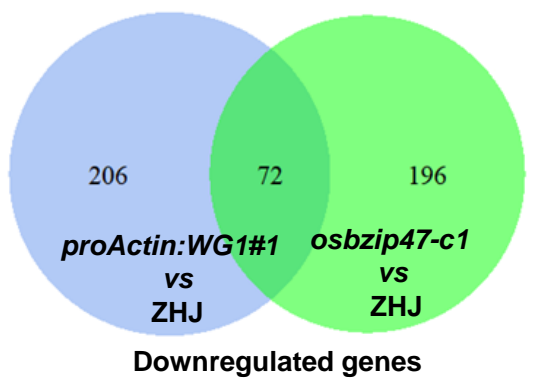

Supplementary Figure 16. Co-regulated genes in proActin:WG1\#1 and osbzip47-c1.

(A, B) Differentially expressed genes (DEGs) in proActin:WG1\#1 and osbzip47-c1 were displayed by Venn diagram. A total of 421 and 180 genes were upregulated in proActin:WG1\#1 (vs ZHJ; blue) and osbzip47-c1 (vs ZHJ; green). 71 genes were upregulated by both proActin:WG1\#1 and osbzip47-c1 (A). While a total of 278 genes were downregulated in proActin:WG1\#1 (vs ZHJ; blue), and 268 genes were downregulated in osbzip47c1 (vs ZHJ; green). 72 genes were downregulated by both proActin:WG1\#1 and osbzip47-c1 (B). vs, versus. 
Supplementary Table 1. List of primers used in this study.

\section{Primers for constructs}

\begin{tabular}{|c|c|}
\hline Construct name & Primers sequences (F: Forward R: Reverse) \\
\hline \multirow{2}{*}{ proACTIN:WG1 } & F: GCAGGAATTCAAGCTTATGCAGTACGGAGCGGCG \\
\hline & R: AGTCACTATGGTCGACCTAGAGCCAGAGCGCGCC \\
\hline \multirow{2}{*}{ proACTIN:OsbZIP47 } & F: GCAGGAATTCAAGCTTATGGCGGCGCACCAGGGGATG \\
\hline & R: AGTCACTATGGTCGACCTAGTCCCTCGGCCGCGCCA \\
\hline \multirow{2}{*}{$\begin{array}{l}\text { Public primer for CRISPR } \\
\text { vector construction }\end{array}$} & F1: GACGGCCAGTGCCAAGCTTCTCGGATCCACTAGTAACGGC \\
\hline & R2: CCTGCAGGCATGCAAGCTTCGACCTCGAGCGGCCGCCAGT \\
\hline \multirow{2}{*}{ CRI-WG1-1 } & F2: GTCATGGCCGCGCACATCAAGTTTTAGAGCTAGAAATAGCA \\
\hline & R1: TTGATGTGCGCGGCCATGACACACAAGCGACAGCGCGCGGG \\
\hline \multirow{2}{*}{ CRI-WG1-2 } & F2: GGAGCAGGCGTGGTACATGCGTTTTAGAGCTAGAAATAGCA \\
\hline & R1: GCATGTACCACGCCTGCTCCACACAAGCGACAGCGCGCGGG \\
\hline \multirow{2}{*}{ CRI-GW2 } & F2: CGCAGGGGCTGTACGAGCACGTTTTAGAGCTAGAAATAGCA \\
\hline & R1: GTGCTCGTACAGCCCCTGCGACACAAGCGACAGCGCGCGGG \\
\hline \multirow{2}{*}{ CRI-OsbZIP47 } & F2: GCACATCAACGACCTGCGGGGTTTTAGAGCTAGAAATAGCA \\
\hline & R1: CCCGCAGGTCGTTGATGTGCACACAAGCGACAGCGCGCGGG \\
\hline \multirow{2}{*}{ proWG1:WG1-GUS } & F: CGGGGCGTAATCTAGAGCTTGAATGGCACGTGACCG \\
\hline & R: GGATCGATCCTCTAGAGAGCCAGAGCGCGCCGGCC \\
\hline \multirow{2}{*}{ proOsbZIP47:OsbZIP47-GUS } & F: CGGGGCGTAATCTAGAAGACATGGTATGTGGTGGGTCCTACCG \\
\hline & R: GGATCGATCCTCTAGAGTCCCTCGGCCGCGCCAGCCACA \\
\hline \multirow{2}{*}{ proWG1:WG1-GFP } & F: CGACTCTAGAGGATCCGCTTGAATGGCACGTGACCGTCTG \\
\hline & R: CCCCCTCGAGGCGCGGAGCCAGAGCGCGCCGGCCTCCTTGAG \\
\hline \multirow{2}{*}{ proOsbZIP47:OsbZIP47-GFP } & F: CGACTCTAGAGGATCCAGACATGGTATGTGGTGGGTCC \\
\hline & R: CCCCCTCGAGGCGCGGTCCCTCGGCCGCGCCAGCCACA \\
\hline \multirow{2}{*}{ pro35S:GFP-GW2 } & F: GATCGGGGAAATTCGAGCTCCTACAACCATGCCAACCCTTGC \\
\hline & R: CTATACAAAGGCGCGATGGGGAACAGGATAGGGGGGA \\
\hline \multirow{2}{*}{ pro35S:GFP-ASP1 } & F: GATCGGGGAAATTCGAGCTCTCAGACTTCTGGTTTGTTAGCT \\
\hline & R: CTATACAAAGGCGCGATGTCGTCGCTTAGCAGGGAG \\
\hline \multirow{2}{*}{ pro35S:Myc-WG1 } & F: CGAAATCGATGGATCCTAATGCAGTACGGAGCGGCGGC \\
\hline & R: GAGCTCACTAGTTAATTAACTAGAGCCAGAGCGCGCC \\
\hline \multirow{2}{*}{ pro35S:Myc-OsbZIP47 } & F: CGAAATCGATGGATCCTAATGGCGGCGCACCAGGGG \\
\hline & R: GAGCTCACTAGTTAATTAACTAGTCCCTCGGCCGCGC \\
\hline \multirow{2}{*}{ pro35S:Myc-GW2 } & F: CGAAATCGATGGATCCTAATGGGGAACAGGATAGGGGGGAG \\
\hline & R: GAGCTCACTAGTTAATTAACTACAACCATGCCAACCCTTGCG \\
\hline \multirow{2}{*}{ cLUC-WG1 } & F: GGGGCGGTACCCGGGGATCCATGCAGTACGGAGCGGCG \\
\hline & R: CGAAAGCTCTGCAGGTCGACCTAGAGCCAGAGCGCGC \\
\hline \multirow{2}{*}{ cLUC-OsbZIP47 } & F: GGGGCGGTACCCGGGGATCCATGGCGGCGCACCAGGG \\
\hline & R: CGAAAGCTCTGCAGGTCGACCTAGTCCCTCGGCCGCGCCAGCCA \\
\hline \multirow{2}{*}{ WG1-nLUC } & F: GAGCTCGGTACCCGGGGATCCATGCAGTACGGAGCGGCG \\
\hline & R: GCGTACGAGATCTGGTCGACGAGCCAGAGCGCGCCGGC \\
\hline \multirow{2}{*}{ GW2-nLUC } & F: GAGCTCGGTACCCGGGGATCCATGGGGAACAGGATAGGG \\
\hline & R: GCGTACGAGATCTGGTCGACCAACCATGCCAACCC \\
\hline \multirow{2}{*}{$A S P 1-n L U C$} & F: GAGCTCGGTACCCGGGGATCCATGTCGTCGCTTAGCAGGGAG \\
\hline & R: GCGTACGAGATCTGGTCGACGACTTCTGGTTTGTTAGCT \\
\hline cYFP-GW2 & F: CGGAAGCGGAGGTGGAGGTAGTATGGGGAACAGGATAGGGG \\
\hline
\end{tabular}




\begin{tabular}{|c|c|}
\hline & R: CGGTGGCGGCCGCTCTAGAACTAGTCTACAACCATGCCAACCCT \\
\hline \multirow{2}{*}{ nYFP-WG1 } & F: CGGAAGCGGAGGTGGAGGTAGTATGCAGTACGGAGCGGCGG \\
\hline & R: CGGTGGCGGCCGCTCTAGAACTAGTCTAGAGCCAGAGCGCGCCG \\
\hline \multirow{2}{*}{$B D-W G 1$} & F: GGACCTGCATATGGCCATGCAGTACGGAGCGGCGGCC \\
\hline & R: TAGTTATGCGGCCGCCTAGAGCCAGAGCGCGCCGGC \\
\hline \multirow{2}{*}{$B D-W G 1 \triangle A L W L$} & F: GGACCTGCATATGGCCATGCAGTACGGAGCGGCGGCC \\
\hline & R: TAGTTATGCGGCCGCCTAGCCGGCCTCCTTG \\
\hline \multirow{2}{*}{$B D-W G 1 m A L W L$} & F: GGACCTGCATATGGCCATGCAGTACGGAGCGGCGGCC \\
\hline & R: TAGTTATGCGGCCGCCTAAGCCCAGGCCGCGCCGGCCTCC \\
\hline \multirow{2}{*}{$B D-A S P 1$} & F: GGACCTGCATATGGCCATGTCGTCGCTTAGCAGG \\
\hline & R: TAGTTATGCGGCCGCTCAGACTTCTGGTTTGTTAG \\
\hline \multirow{2}{*}{$A D-W G 1$} & F: CCATGGAGGCCAGTGAAATGCAGTACGGAGCGGCGGCCGAG \\
\hline & R: TTCATCTGCAGCTCGCTAGAGCCAGAGCGCGCCGGCCT \\
\hline \multirow{2}{*}{$A D-G W 2$} & F: CCATGGAGGCCAGTGAAATGGGGAACAGGATAGGGGGGA \\
\hline & R: TTCATCTGCAGCTCGCTACAACCATGCCAACCCTTG \\
\hline \multirow{2}{*}{$A D-G W 2(1-114)$} & F: CCATGGAGGCCAGTGAAATGGGGAACAGGATAGGGGGGA \\
\hline & R: TTCATCTGCAGCTCGCTAACGATACTCCACAGCAT \\
\hline \multirow{2}{*}{ AD-GW2 (94-425) } & F: CCATGGAGGCCAGTGAAATGCACACTGCTCAGCCTACA \\
\hline & R: TTCATCTGCAGCTCGCTACAACCATGCCAACCCTTG \\
\hline \multirow{2}{*}{$A D-O s b Z I P 47$} & F: CCATGGAGGCCAGTGAAATGGCGGCGCACCAGGGGA \\
\hline & R: TTCATCTGCAGCTCGCTAGTCCCTCGGCCGCGCCAG \\
\hline \multirow{2}{*}{$A D-O s b Z I P 47(1-161)$} & F: CCATGGAGGCCAGTGAAATGGCGGCGCACCAGGGGA \\
\hline & R: TTCATCTGCAGCTCGCTAACCATTTCCTCCG \\
\hline \multirow{2}{*}{ AD-OsbZIP47 (139-385) } & F: CCATGGAGGCCAGTGAAATGAGACAGCAGGGAAT \\
\hline & R: TTCATCTGCAGCTCGCTAGTCCCTCGGCCGCGCCAG \\
\hline \multirow{2}{*}{$A D-A S P 1$} & F: CCATGGAGGCCAGTGAAATGTCGTCGCTTAGCAGGGAG \\
\hline & R: TTCATCTGCAGCTCGTCAGACTTCTGGTTTGTTAGCT \\
\hline \multirow{2}{*}{$M B P-G W 2$} & F: TTCAGAATTCGGATCCATGGGGAACAGGATAGGGGGGAG \\
\hline & R: CGACTCTAGAGGATCCCTACAACCATGCCAACCCTTG \\
\hline \multirow{2}{*}{ FLAG-WG1 } & F: CGCTGGATCCGAATTCATGCAGTACGGAGCGGCG \\
\hline & R: GGTGGTGGTGCTCGACTAGAGCCAGAGCGCGCC \\
\hline \multirow{2}{*}{ WG1-HIS } & F: TATACATATGGCTAGATGCAGTACGGAGC \\
\hline & R: GGTGGTGGTGCTCGAGGAGCCAGAGCGCGCC \\
\hline \multirow{2}{*}{ FLAG-OsbZIP47 } & F: CCAGATTACGCTGGATCCGAATTCATGGCGGCGCACCAGGGGA \\
\hline & R: AGTGGTGGTGGTGGTGGTGCTCGAGCTAGTCCCTCGGCCGCGCC \\
\hline \multirow{2}{*}{$B D-O s b Z I P 47$} & F: CCCCCGGGCTGCAGGAATTCATGGCGGCGCACCAGGGGAT \\
\hline & R: CCCCCTCGAGGTCGACCTAGTCCCTCGGCCGCGCCA \\
\hline \multirow{2}{*}{$35 S-W G 1$} & F: CCCCCGGGCTGCAGGAATTCATGCAGTACGGAGCGGCGGC \\
\hline & R: CCCCCTCGAGGTCGACCTAGAGCCAGAGCGCGCC \\
\hline \multirow{2}{*}{$35 S-W G 1 \triangle A L W L$} & F: CCCCCGGGCTGCAGGAATTCATGCAGTACGGAGCGGCGGC \\
\hline & R: CCCCCTCGAGGTCGACCTAGCCGGCCTCCTTGAG \\
\hline \multirow{4}{*}{ BD-OsbZIP47(C17/197/269S) } & F: CCCCCGGGCTGCAGGAATTCATGGCGGCGCACCAGGGGAT \\
\hline & R: CCCCCTCGAGGTCGACCTAGTCCCTCGGCCGCGCCA \\
\hline & GAL4-AD-F: TCCGAACCTCATAACAACTC \\
\hline & F1: CCGGTTCTCCCTGCCGAGGATG \\
\hline
\end{tabular}


R1: CATCCTCGGCAGGGAGAACCGG

F2: AGCGACGACGAGCTGTCCGAG

R2: CTCGGACAGCTCGTCGTCGCT

F3: GCTGATGGGCATCTCCAACCTGCAGCAGTC

R3: GACTGCTGCAGGTTGGAGATGCCCATCAGC

\begin{tabular}{|c|c|}
\hline \multicolumn{2}{|r|}{ Primers for identification of mutants } \\
\hline Mutant name & Primers (F: Forward R: Reverse) \\
\hline \multirow{2}{*}{$w g 1-1 / w g 1-2 / w g 1-3$} & F: GGCATTCTACAATCCCATCGT \\
\hline & R: AGCAGCAGCAGCAGGTAA (sequencing) \\
\hline \multirow{2}{*}{$w g 1-1$} & F: TGCATGTGCCACGCCGTGAA \\
\hline & R: CTAAGCCAGAGCACGCCAAT \\
\hline \multirow{2}{*}{$g w 2-c 1$} & F: GGTGGAGGAGAGGTACACGA \\
\hline & R: CGGTTTTATACCAGGAAGCAG \\
\hline \multirow{2}{*}{ OsbZIP47-c1/2 } & F: CAGGTACGTTGGCGTTCGAC \\
\hline & R: GTTGCTGAATTGGGTGGGTGAT \\
\hline \multirow{2}{*}{ asp1-101 } & F: ATGTCCGTGTTGACGAGG \\
\hline & R: TCAGACTTCTGGTTTGTTAG \\
\hline \multicolumn{2}{|r|}{ Primers for quantitative RT-PCR } \\
\hline Gene name & Primers (F: Forward R: Reverse) \\
\hline \multirow{2}{*}{ ACTIN1 } & F: TGCTATGTACGTCGCCATCCAG \\
\hline & R: AATGAGTAACCACGCTCCGTCA \\
\hline \multirow{2}{*}{ WG1 } & F: AGCAGGCGTGGTACATG \\
\hline & R: TTCACGGCGTGGCACAT (for Figure S1E) \\
\hline \multirow{2}{*}{ WG1 } & F: TCGTCTTCATCGGCGGCAAG \\
\hline & R: CAGCAGCAGCAGGTAAAGC (for Figure 2B) \\
\hline \multirow{2}{*}{ OsbZIP47 } & F: GCCAACGTACCATTTCCTCC \\
\hline & R: GAAGAGCCGGATGAGGAAGA \\
\hline \multirow{2}{*}{ WG1-GFP } & F: ACATCAACGGCTCCCTCGT \\
\hline & R: AGAATTGGGACAACTCCAGTGA \\
\hline
\end{tabular}

